\title{
A Dynamic Multiobjective Evolutionary Algorithm Based on A Dynamic Evolutionary Environment Model
}

\author{
Juan Zou ${ }^{\mathrm{a}, *}$, Qingya $\mathrm{Li}^{\mathrm{a}}$, Shengxiang Yang ${ }^{\mathrm{c}}$, Jinhua Zheng ${ }^{\mathrm{a}, \mathrm{b}}$, Zhou Peng ${ }^{\mathrm{a}}$, \\ Tingrui Pei ${ }^{\mathrm{a}}$ \\ ${ }^{a}$ Key Laboratory of Intelligent Computing and Information Processing (Ministry of \\ Education), Xiangtan University, Xiangtan 411105, China \\ ${ }^{b}$ Hunan Provincial Key Laboratory of Intelligent Information Processing and Application, \\ Hengyang Normal University, Hengyang 421002, China \\ ${ }^{c}$ School of Computer Science and Informatics, De Montfort University, Leicester LE1 9BH, \\ U.K.
}

\begin{abstract}
Traditional dynamic multiobjective evolutionary algorithms usually imitate the evolution of nature, maintaining diversity of population through different strategies and making the population track the Pareto optimal solution set efficiently after the environmental change. However, these algorithms neglect the role of the dynamic environment in evolution, leading to the lacking of active guided search. In this paper, a dynamic multiobjective evolutionary algorithm based on a dynamic evolutionary environment model is proposed (DEE-DMOEA). When the environment has not changed, this algorithm makes use of the evolutionary environment to record the knowledge and information generated in evolution, and in turn, the knowledge and information guide the search. When a change is detected, the algorithm helps the population adapt to the new environment through building a dynamic evolutionary environment model, which enhances the diversity of the population by the guided method, and makes the environment and population evolve simultaneously. In addition, an implementation of the algorithm about the dynamic evolutionary environment model is introduced in this paper. The environment area and the unit area are employed to express
\end{abstract}

\footnotetext{
${ }^{*}$ Corresponding author

Email addresses: zoujuan@xtu.edu.cn (Juan Zou), smileliqingya@gmail.com (Qingya Li), syang@dmu.ac.uk (Shengxiang Yang), jhzheng@xtu.edu.cn (Jinhua Zheng), zpeng@xtu.edu.cn (Zhou Peng), peitingrui@xtu.edu.cn (Tingrui Pei)
}

Preprint submitted to Swarm and Evolutionary Computation 
the evolutionary environment. Furthermore, the strategies of constraint, facilitation and guidance for the evolution are proposed. Compared with three other state-of-the-art strategies on a series of test problems with linear or nonlinear correlation between design variables, the algorithm has shown its effectiveness for dealing with the dynamic multiobjective problems.

Keywords: Dynamic multiobjective optmization, evolutionary algorithms, evolutionary environment, dynamic evolutionary environment model

\section{Introduction}

Many real-world problems are dynamic multiobjective optimization problems (DMOPs), with conflicts among multiple objectives as well as objective functions that change over time [1]. Tracking the Pareto optimal solution set after a change is an important and challenging issue. On these issues, the researched objectives often change intricately with time. The goal of the traditional evolutionary algorithms is to make the population gradually converge to get a satisfactory solution set, but this makes the population lose diversity. Especially, in the later stages of the evolution, the population will gradually lose the ability to adapt to the environmental changes, which is a challenge of the traditional evolutionary algorithms in the dynamic environment [2, 3, 24, 4, 5]. In order to track the optimal solution set in a timely manner after a change, researchers need to make some adjustments on the traditional static multiobjective algorithms 6 , 7, 86, 9], so that they can quickly respond to the environmental changes.

In recent years, researchers have designed many new ways to solve DMOPs on the basis of static algorithms 10,11, 13, 14, 16, 17, 18, 19, 20, 21|, such as random initialization 12, 25, 26, 18, 17], hyper mutation 25, 22, 15, 33], memory [25, 26, 29, 30, 36, 23, 41] , and prediction [31, 32, 33, 34, 35, 36, 42, 48, 49]. These strategies have been proven effective for solving DMOPs; however, they are defective in the following ways. Firstly, random initialization, hyper mutation and dynamic migration strategies are all a blind way to enhance population 
diversity without a right guidance, and the performance of convergence is unsatisfactory when dealing with complex DMOPs. Secondly, memory strategy reuses the optimal solutions which are previously searched in the previous time to rapidly respond to changes in the new environment. This strategy can achieve good results for periodic problems. However, for non-periodic problems or in the first cycle of the changing environment, population is still in the process of blind evolution. Thus, the algorithm is difficult to obtain a good convergence. Lastly, methods that are based on prediction generate a new optimal solution set by the prediction model for the evolution of the population, and help the algorith$\mathrm{m}$ to respond quickly to new changes. However, obtaining accurate predictions remains a primary difficulty. Thus, designing a more accurate prediction model is still a focus of the present research.

To solve these problems, on the premise of less history information and utilizing the characteristics of the evolutionary environment itself, the paper proposes a dynamic multiobjective evolutionary algorithm based on a dynamic environment evolutionary model, referred to as DEE-DMOEA. Current dynamic multiobjective optimization algorithms do not consider the role of the dynamic environment for the evolutionary population. Actually, the effect of the environment on evolutionary individuals is very important, for individuals must survive and evolve in a specific environment. The wonderful interaction between the natural environment and the biology that makes biomass have such a present perfect structure. Therefore, how to research from the perspective of the dynamic environment, using the dynamic environmental knowledge to guide the evolution of population in the new environment to accelerate convergence of the population is the research focus in this paper.

The rest of the paper is organized as follows. Section 2 provides important terminology. Section 3 describes the dynamic environment evolutionary model. Section 4 describes the implementation of the evolutionary model. Section 5 introduces the test problems and evaluation metrics. Section 6 gives the experimental results and analysis. Section 7 provides the conclusions and future work. 


\section{Background}

A minimization problem is considered here without loss of generality. The dynamic multiobjective optimization problem [1] can be described as:

$$
\left\{\begin{array}{l}
\min _{x \in \Omega} F(x, t)=\left(f_{1}(x, t), f_{2}(x, t), \ldots, f_{m}(x, t)\right)^{T} \\
\text { s.t. } g_{i}(x, t) \leq 0 i=1,2, \ldots, p ; h_{j}=0 j=1,2, \ldots, q
\end{array}\right.
$$

where $t$ is the time variable and $x=\left(x_{1}, x_{2}, \ldots, x_{n}\right)$ is the $\mathrm{n}$-dimensional decision vector bounded by the decision space $\Omega . F=\left(f_{1}, f_{2}, \ldots, f_{m}\right)$ presents the set of $m$ objectives to be minimized and the functions of $g_{i} \leq 0 i=1,2, \ldots, p$ and $h_{j}=0 j=1,2, \ldots, q$ present the set of inequality and equality constraints.

Definition 1 (Pareto Dominance). $p$ and $q$ are any two individuals in the population; $p$ is said to dominate $q$, denoted by $f(p) \prec f(q)$ iff $f_{i}(p) \leq f_{i}(q)$ $\forall i=\{1,2, \ldots, m\}$ and $f_{j}(p)<f_{j}(q) \exists j \in\{1,2, \ldots, m\}$.

Definition 2 (Pareto Optimal Set(PS)). $x$ is the decision variable; $\Omega$ is the decision space; $F$ is the objective function; thus, the PS [n] is the set of all non-dominated solutions and is defined mathematically as:

$$
P S:=\left\{x \in \Omega \mid \nexists x^{\star} \in \Omega, F\left(x^{\star}\right) \prec F(x)\right\}
$$

Definition 3 (Pareto Optimal Front $(\mathbf{P F})) \cdot x$ is the decision variable; $F$ is the objective function; thus, the PF [r] is the set of non-dominated solutions with respect to the objective space and is defined mathematically as:

$$
P F:=\{y=F(x) \mid x \in P S\}
$$

\section{Dynamic Environment Evolutionary Model}

In ecology, environment refers to external matters such as the surrounding ecosystem which affects biological communities. In our dynamic environment evolutionary model, the environment refers to a group of entities which can guide and promote the evolution of the population. Especially, after environmental 
changes, it can guide the evolution and convergence of the population in the new environment.

An evolutionary population must survive and evolve in a specific environment. The environment plays constraint, facilitating and guiding roles for the evolution of the population, and these three environmental roles are completely different. Constraint is mainly used to ensure the legitimacy of individuals; facilitating is mainly used to enhance the efficiency of the evolution and improve the distribution of evolutionary population. Guiding is mainly used to help the population adapt to the new environment. At the same time, the evolutionary population is counteractive to the evolutionary environment, which is mainly shown in the impact on the attributes of the evolutionary environment, such as the changes of the current evolutionary state and the update of the environmental knowledge.

In a dynamic environment, how to maintain the diversity of the population after an environmental change is the key to solve DMOPs. When the environment changes, environmental information and knowledge make a difference. Making full use of this information in a dynamic environment to help the population adapt to the new environment plays an important role for solving DMOPs.

Fig. 1 shows a general framework of a dynamic evolutionary environment model. A dynamic environment model consists of two different kinds of environment before and after the environmental change. Environment elements include environmental knowledge, environmental evaluation, environmental constraint before change and environmental regulation after change. Among them, environmental knowledge can be divided into static knowledge and dynamic knowledge. Static knowledge is the preset environmental attributes which maintain constant values in the process of evolution, such as environmental capacity and dimensions. Dynamic knowledge is the environmental attributes which are affected by population in the process of environmental change, such as the congestion degree, the domain to be oriented, direction of environmental change and newly generated individuals for guiding evolution. the environmental eval- 
uation mechanism evaluates the living conditions of the population or individuals according to environmental knowledge, such as individual location in the environment and the entire population distribution.

Environmental constraint before change mainly includes two parts: 1) the satisfaction constraint of the expected solution set, and 2) the distribution constraint of the expected solution set. Environmental constraint mostly reflects on the guidance for the population, that is to say, it can achieve the evolution in the environment by environmental constraint.

Environmental regulation after change means that individuals need to make the corresponding change in order to adapt to the new environment. There are two different kinds of environment exchange information to facilitate and guide the evolution of the population. In return, the population will send the feedback information which is generated in the process of evolution to the environment, updating the environmental knowledge and achieving co-evolution.

The dynamic environmental facilitating and guiding mechanism for the population is the core of DEE-DMOEA, which determines the evolutionary direction of the population and plays a decisive motivational role in the evolution. The dynamic environmental facilitating mechanism indicates that, when the environment does not change, on the one hand, it promotes the individual accelerated evolution in compliance with environmental satisfaction constraints. On the other hand, it balances the density of population distribution and expands the range of population distribution in compliance with the environmental distribution constraints. The dynamic environmental guiding mechanism aims to enhance population diversity by guided method according to environmental regulation after change, help population adapt to the new environment, and accelerate the algorithm to quickly track the new Pareto optimal solution set.

\section{Implementation of The Evolutionary Model}

Each individual in a dynamic environment has a living space. Here we use a mechanism which is similar to the grid, referred to as the environment 


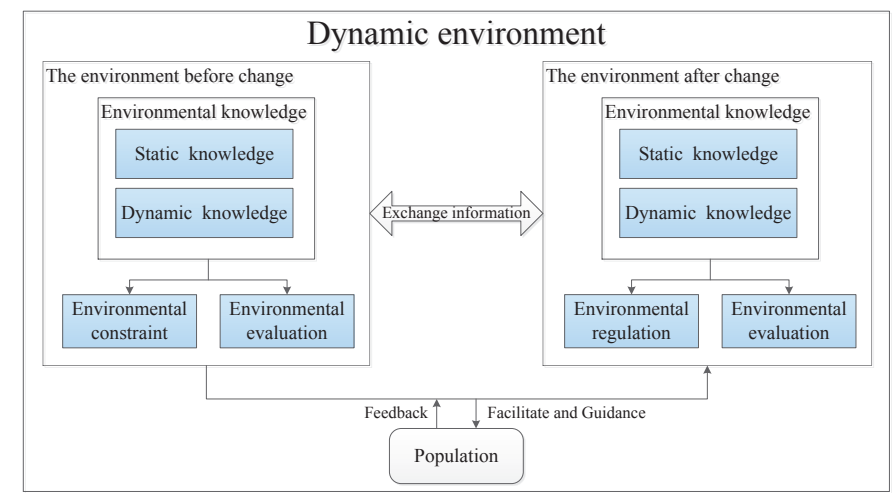

Figure 1: A general framework of dynamic environment evolutionary model.

domain, to store the individuals in the dynamic environment. The environment domain consists of many identical grids, which are called the unit domains. The dimensions of the environment domain and unit domains are the same as the objective dimensions. The position of an individual in the environment domain will change accordingly when the environment changes. Therefore, in a dynamic environment, as shown in Fig. 2, when the environment does not change, the range of environment domain and the size of unit domain are determined by the location and distribution of population in the environment; the environment domain will constantly adjust with the evolution of the population. When the environment changes, the range of environment domain and the size of unit domain are co-determined by the different distributions of the population before and after the environmental change.

Bottom and top boundaries of each dimension in the environment domain are calculated as follows:

$$
\begin{aligned}
l b_{i} & =\min \left(P_{i}\right)-\left(\max \left(P_{i}\right)-\min \left(P_{i}\right) /(2 \times n u m)\right) \\
u b_{i} & =\max \left(P_{i}\right)+\left(\max \left(P_{i}\right)-\min \left(P_{i}\right) /(2 \times n u m)\right)
\end{aligned}
$$

where num is the number of unit domains on each dimension in the objective space. The higher the objective dimension, the smaller the value of num. For example, num can be set to 40 for two objectives and can be set to 10 for three 


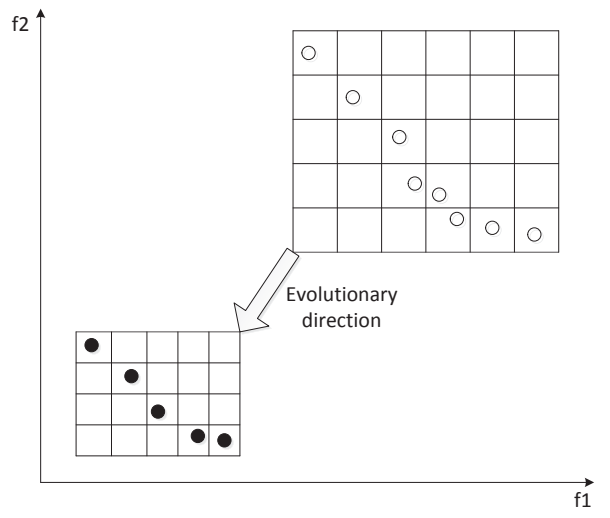

(a) The environment before change

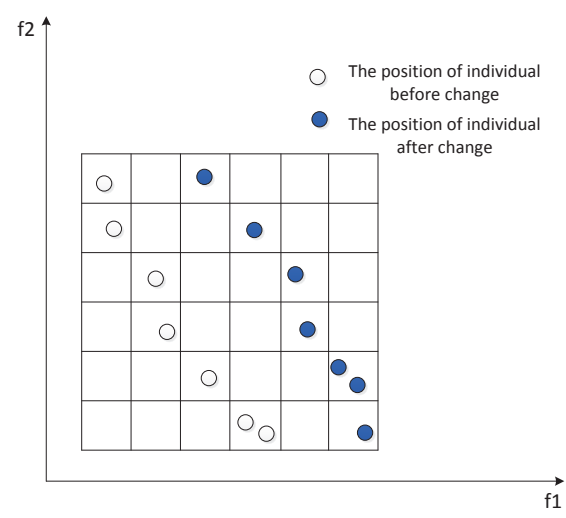

(b) The environment after change

Figure 2: Two different types of environment.

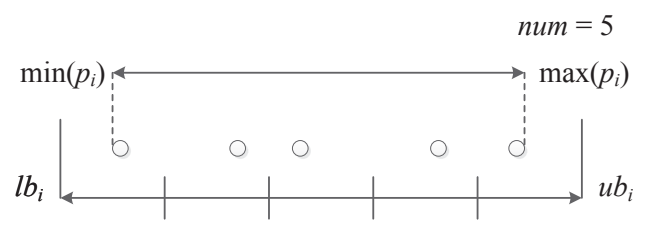

Figure 3: The environment domain set on i-dimensional objective.

objectives. When the environment does not change, $\min \left(P_{i}\right)$ and $\max \left(P_{i}\right)$ denote the minimum and maximum values of the $i$ th objective of population $P$. While a change is detected, $\min \left(P_{i}\right)$ and $\max \left(P_{i}\right)$ denote the minimum and maximum values of the $i$ th objective of population $P$ in the two different kinds of environments before and after change, namely $\min \left(P . o l d F_{i}, P . n e w F_{i}\right)$ and $\max \left(P . o l d F_{i}, P . n e w F_{i}\right)$. As shown in Fig. 3, the size of unit domain on the $i$ th objective is area_size $e_{i}=\left(u b_{i}-l b_{i}\right) /$ num.

In a dynamic environment, when the environment does not change, each individual belongs to a specific unit domain. We denote indiv.area as the unit domain to which individual indiv belongs. According to the boundary of the environment domain and the size of unit domain, the unit domain position (domain coordinate) of each individual can be determined. The domain coordinate 
of indiv.area on the $i$-th objective dimension can be calculated as Eq. (2).

$$
\text { indiv.area }_{i}=\left\lfloor\left(\text { indiv. } F_{i}-l b_{i}\right) / \text { area_size }_{i}\right\rfloor
$$

where $i n d i v . F_{i}$ is the $i$-th objective value of individual indiv. In Eq. (1) of calculating $l b_{i}, \min \left(P_{i}\right)$ and $\max \left(P_{i}\right)$ denote the minimum and maximum values of the $i$ th objective of population $P$.

While the environment changes, each individual may belong to two different unit domains before and after the environmental change, at this time the environment domain will be reconstructed. Therefore, we denote indiv.old_area as the unit domain to which individual indiv belongs before the environmental change and indiv.new_area as the unit domain to which individual indiv belongs after the environmental change. The domain coordinates of indiv.old_area and indiv.new_area on the $i$-th objective can be calculated as follows:

$$
\begin{aligned}
\text { indiv.old_area }_{i} & =\left\lfloor\left(\text { indiv.old }_{i}-l b_{i}\right) / \text { area_size }_{i}\right\rfloor \\
\text { indiv.new_area }_{i} & =\left\lfloor\left(\text { indiv.new } F_{i}-l b_{i}\right) / \text { area_size } e_{i}\right\rfloor
\end{aligned}
$$

where indiv.old $F_{i}$ and indiv.new $F_{i}$ are respectively the $i$-th objective values before and after an environmental change. In Eq. (1) of calculating $l b_{i}, \min \left(P_{i}\right)$ and $\max \left(P_{i}\right)$ denote the minimum and maximum values of the $i$-th objective of population $P$ in the two different kinds of environments before and after a change.

The environment domain and unit domain have been set, then the various elements of composing dynamic environment and their implementation will be introduced.

\subsection{Environmental Knowledge}

Environmental knowledge is an important part of the environment, which denotes the information recorded in the current dynamic environment. In our approach, environmental knowledge is divided into two types: the environment domain knowledge and unit domain knowledge. The environment domain knowledge is divided into static and dynamic environment domain knowledge. Static 
environment domain knowledge includes environmental capacity, the number of unit domains on each dimension and other preset environmental attributes. Dynamic environment domain knowledge includes the bottom boundary and top boundary of the environment domain on each dimension, the size of unit domain, the number of unit domains containing any individuals, the domain to be oriented, the direction of environmental change, the newly generated individuals for guiding the evolution, and other environmental attributes which are affected by the population. The new individuals are a series of re-initialized individuals to help the population adapt to the new environment after an environmental change and accelerate the convergence of the population and individuals, which will be described in detail in Section 4.3.

Unit domain knowledge is dynamic knowledge, which includes the number of individuals in each unit domain, a representative individual and the nondominated unit domains. Representative individual is the optimal individual in a unit domain. Here, we set the individual with the nearest Euclidean distance to the origin of unit domain as the representative individual. The origin of the unit domain is the minimum on each dimension.

\subsection{Environmental Constraint}

When the environment does not change, survival and evolution of each individual in the environment domain are required to meet the satisfaction constraint; not all offspring generated by evolution can enter the environment domain. Here, we stipulate that the individual in the environment domain must satisfy the following two constraints:

1) Individuals in each unit domain of the environment domain are mutually non-dominated.

2) Unit domains in the environment domain must be mutually domain strong non-dominated. The unit domain here refers to the unit domain containing any individual. 
3) The strong dominance relation is stricter than the Pareto dominance. The domain strong dominance relation is defined as follows.

Definition 4 (Domain strong dominance). $A$ and $B$ are any two unit domains in the environment domain; $A$ is said to domain strong dominate $B$, denoted by $A \prec \prec_{\text {area }} B$ if ${\text { A. } \text { area }_{i}<B \text {. area }}_{i} \forall i=\{1,2, \ldots, r\}$. Where $r$ is the dimensions of the unit domain.

Similarly, the domain dominance can be defined as follows:

Definition 5 (Domain dominance). $C$ and $D$ are any two unit domains in the environment domain, $C$ is said to domain dominate $D$, denoted by $C \prec$ area

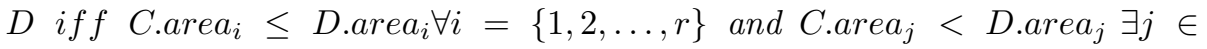
$\{1,2, \ldots, r\}$.

\subsection{Environmental Evaluation}

In a dynamic evolutionary environment model, the evaluation mechanism needs to evaluate not only the fitness of the population, but also the living conditions of the population and individuals according to environmental knowledge, and prepares for guiding evolution. The evaluation mechanism is divided into two types, one is evaluation for the individual, and the other is evaluation for the population.

Evaluation for the individual is to calculate the unit domain coordinates for each individual when the environment does not change according to Eq. (2), and to determine the representative individual. While the environment changes, the evaluation calculates two different unit domain coordinates for each individual, and provides feedback to the environment to construct a new dynamic environment.

Evaluation for the population first evaluates the distribution of the entire population in the new environment according to the environmental knowledge, then generates a new series of guide-individuals to prepare for guiding evolution. 
The new guide-individuals are defined by Eq. (4):

$$
\begin{aligned}
& \text { init }_{k}^{t}=x_{k}^{t}+\mid\left(C_{k}^{t}-C_{k}^{t-1}\right) \text { Gaussian } \mid, \text { if } C_{k}^{t}-C_{k}^{t-1}>0 \\
& \text { init } t_{k}^{t}=x_{k}^{t}-\mid\left(C_{k}^{t}-C_{k}^{t-1}\right) \text { Gaussian } \mid, \text { if } C_{k}^{t}-C_{k}^{t-1}<0
\end{aligned}
$$

where $x_{k}^{t}$ is the individual at time $\mathrm{t} ; k=1,2, \ldots, n ; n$ is the dimensions of the decision space. Gaussian is a random number generated from a standard normal distribution with mean 0 and variance 1, which has been verified in [27] to be a good strategy to enhance the ability of elaborate search. $C_{k}^{t}$ is the center of non-dominated solutions obtained at time $t$, which can be defined by Eq. (5):

$$
C_{k}^{t}=\frac{1}{\left|P_{N-\text { dominance }}^{t}\right|} \sum_{x_{k}^{t} \in P_{N-\text { dominance }}^{t}} x_{k}^{t}
$$

where $\left|P_{N \text {-dominance }}^{t}\right|$ is the size of non-dominated solutions.

Similarly, the domain coordinates of new guide-individuals are also calculated.

In this way, we use the possible correlation between environmental changes to produce a series of guide-individuals. These individuals will be served as the alternative individuals in the process of environmental facilitating and guiding, to help the population adapt to the new environment and accelerate the convergence of population to the new PF.

\subsection{Environmental Regulation}

In a dynamic environment, different problems have different regulations. The location and distribution of the population in the new environment domain may not be suitable for its evolution and convergence. Therefore, the population needs to make the corresponding change in order to adapt to the new environment.

As shown in Fig. [ just like people's psychological reactions in real life, some individuals want to return to the past environment and continue to survive and evolve, considering that the environment before change is more conducive for evolution. While some individuals do not want to return to the past environment, at the same time they are also confused about where they should go. 


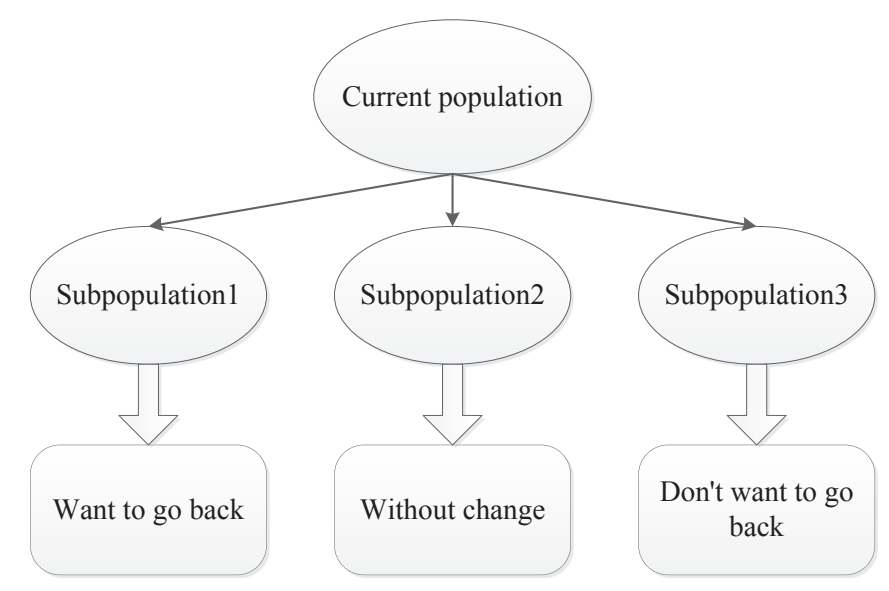

Figure 4: The division of population.

There is also a group of individuals who do not want to make any change, they consider that the current environment is an ideal evolutionary environment.

Therefore, we need to divide the current population into three sub-populations according to the different behavioral characteristics of individuals when the environment changes. Meanwhile, in order to maintain the distribution of subpopulations and avoid crowding the solution set, the three sub-populations need to be more evenly divided. Sub-populations are divided as follows (illustrated by the example of two objectives):

The sizes of three sub-populations are respectively set to num_sub1, num_sub2 and num_sub3 (Initially, for two objectives: 30, 40 and 30; for three objectives: $60,80$ and 60$)$.

For the subpopulation2 which is without any change: we gather directly num_sub2 non-dominated individuals whose crowding-distance [44] is the largest from the original population to subpopulation2.

For the subpopulation1 which wants to go back and the subpopulation3 which does not want to go back: Algorithm 1 gives a detailed procedure of this strategy, where the domain-adjacent is defined as follows:

Definition 6 (Domain-adjacent). $U$ and $V$ are any two individuals in the 


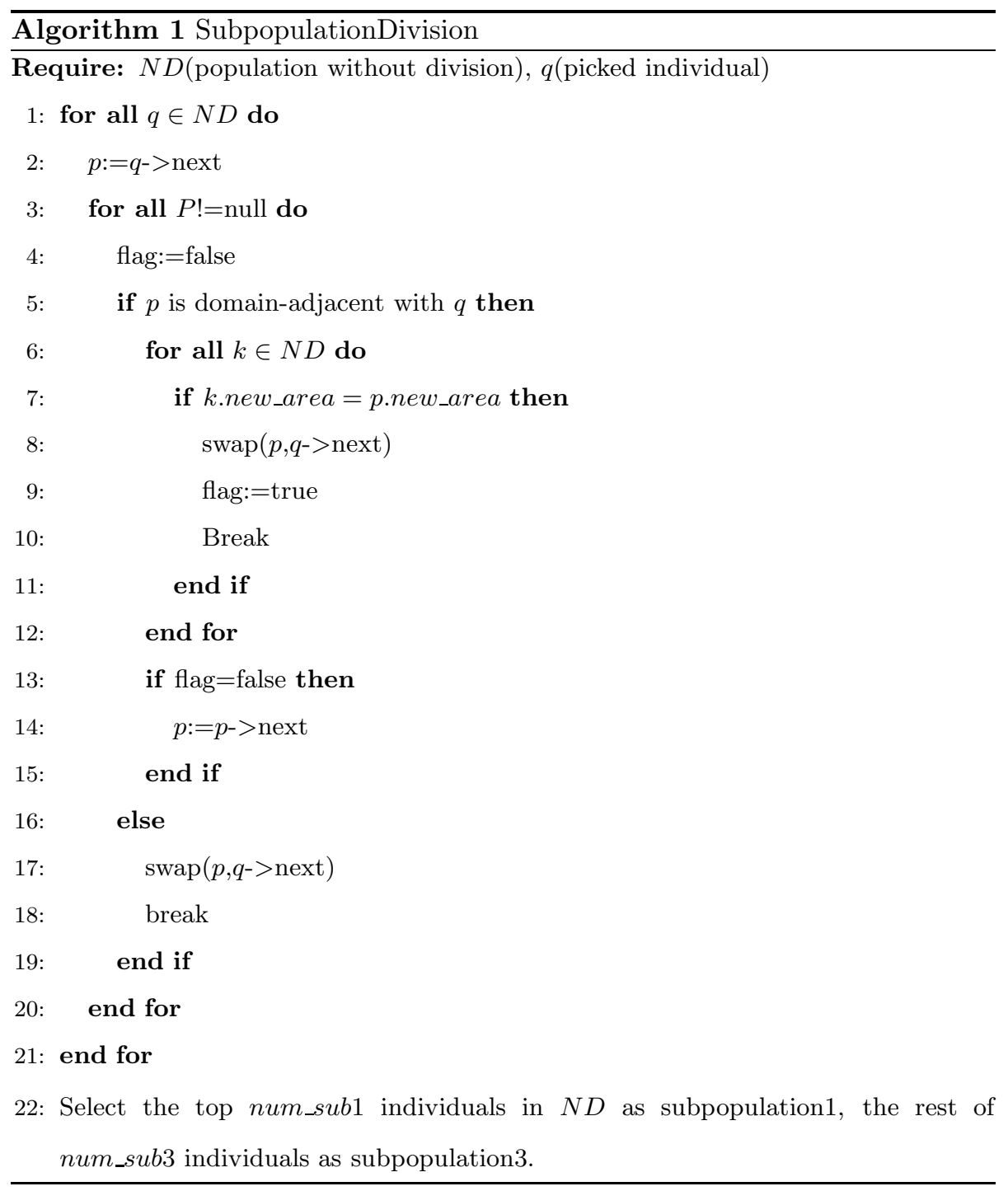




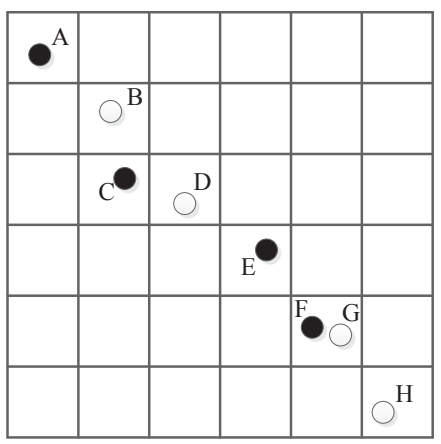

Figure 5: An example about division of the sub-populations.

environment domain; $U$ is domain-adjacent with $V$, if $f \mid U$.new_area $a_{i}-V . n e w \_a r e a_{i} \mid \leq$ min_diff $(i)$. min_diff $(i)$ is the minimum difference on each dimension between any two unit domain coordinates. The unit domain here refers to the unit domain containing any individuals.

Fig. 5 is an example about division of the sub-populations. The first individual A is selected, and then the second individual to compare with $\mathrm{A}$ is selected. The second individual is assumed to be B. Since B is domain-adjacent with $\mathrm{A}$, and its unit domain does not include multiple individuals Therefore, B is discarded. Next, select the individual $\mathrm{C}$, and $\mathrm{C}$ is not domain-adjacent with $\mathrm{A}$, so $\mathrm{C}$ will be divided into the same sub-population with $\mathrm{A}$ and serves as the next individual for comparison. Similarly, $\mathrm{E}$ is not domain-adjacent with $\mathrm{C}$ and is divided into the same sub-population. Despite the fact that $\mathrm{F}$ is domainadjacent with $\mathrm{E}$, its unit domain includes another individual $\mathrm{G}$, so $\mathrm{F}$ will be divided into the same subpopulation with $\mathrm{A}, \mathrm{C}$ and $\mathrm{E}$. Thus, the division ends. A, C, E and F are divided into the same sub-population. B, D, G and $\mathrm{H}$ are divided into another subpopulation.

It is worth noting that environmental regulation in this paper is clearly different from the random division of sub-populations such as charged PSO [37]. Environmental regulation considers the characteristics of different subpopulations to adapt to different environmental changes, and at the same time, 
takes into account the distribution of the solution set, digging and using the environmental knowledge to guide the evolution.

\subsection{Environmental Facilitating Mechanism}

When the environment does not change, on the one hand, the environment facilitating mechanism promotes the individual accelerated evolution in compliance with environmental satisfaction constraints. On the other hand, it balances the density of population distribution and expands the range of population distribution in compliance with environmental distribution constraints. First, we introduce the accelerating action to promote evolution of the population. Classic multiobjective evolutionary algorithms typically recombine by randomly selecting two or more individuals to achieve the evolution of population. However, this simple random selection will be hindered by the evolution to a certain extent. While two different individuals (especially non-dominated individuals) may generate far better offspring than parents which combines advantages of both parents after recombination. In the dynamic environment evolutionary model, we select more efficient individuals to recombine by giving the unit domain a relative fitness assignment. Relative to the unit domain $A_{x_{1}, x_{2}, \ldots, x_{r}}$, the relative fitness of unit domain $B_{y_{1}, y_{2}, \ldots, y_{r}}$ is given as follows:

$$
f(B)_{A}=\sum_{i=1}^{r} \Phi\left(x_{i}, y_{i}\right)
$$

where $x_{i}$ and $y_{i}$ are respectively the $i$ th dimensional domain coordinates, and the definition of function $\Phi$ is given as follows:

$$
\Phi\left(x_{i}, y_{i}\right)= \begin{cases}1 /\left(2+y_{i}-x_{i}\right) & x_{i} \leq y_{i} \\ x_{i}-y_{i} & x_{i}>y_{i}\end{cases}
$$

Relative fitness is a relative concept, it does not represent the pros and cons of units in the environment domain. Relative to the unit domain $A$, the large relative fitness of unit domain $B$ just indicates that selecting the individual in unit domain $B$ and the individual in unit domain $A$ to recombine will generate more excellent offspring than the parents. For instance, in Fig. 6] relative to the 


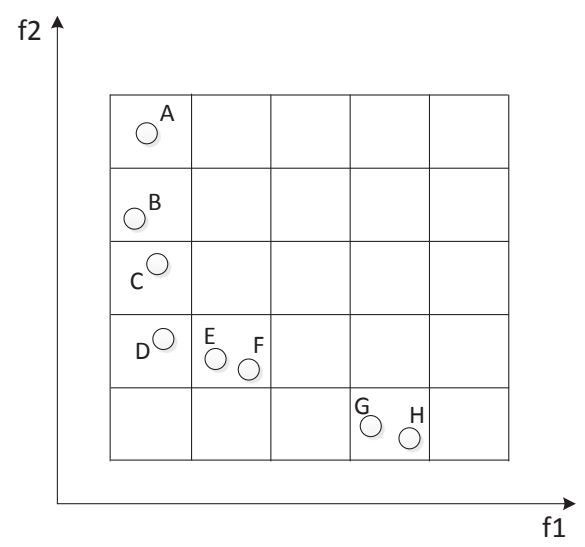

Figure 6: An illustration of individuals in the environment in a bi-objective space.

unit domain $A r e a_{0,2}$, the relative fitness of domain $A r e a_{0,4}, A r e a_{0,3}, A r e a_{0,1}$, Area $_{1,1}$ and Area $_{3,0}$ are respectively 3/4,5/6,3/2,4/3 and 11/5. The domain coordinates of individual $\mathrm{A}$ in unit domain $\mathrm{Area}_{0,4}$ is equal to the individual $\mathrm{C}$ on the objective $f_{1}$, but two units larger on the objective $f_{2}$. So, it is difficult to generate a much better individual than $\mathrm{C}$ in the process of recombination. That is to say, the promoting effect of $\mathrm{A}$ to $\mathrm{C}$ is not obvious. However, the domain coordinates of individual $\mathrm{G}$ or $\mathrm{H}$ in unit domain Area $_{3,0}$ is three units larger than the individual $\mathrm{C}$ on the objective $f_{1}$, but two units smaller on the objective $f_{2}$. So, the generated offspring may inherit the different advantages of parents, that is to say, the promoting effect of $\mathrm{G}$ or $\mathrm{H}$ to $\mathrm{C}$ is very powerful. For an individual to be recombined, we first select a unit domain according to the relative fitness by roulette, and then randomly select an individual within this unit to recombine with it. Here, we choose the SBX [38] and DE [39] operators to promote evolution.

Next, we introduce the balancing and expanding action to population distribution. The balancing and expanding action is mainly implemented by domain orientation. Domain orientation refers to generating new individuals in the domain to be oriented, and meet the environmental distribution constraint. Here, we define the domain to be oriented as follows: 
Definition 7 (The domain to be oriented). $A_{x_{1}, x_{2}, \ldots, x_{r}}$ is the unit domain which has no individual, $A_{x_{1}, x_{2}, \ldots, x_{r}}$ is the domain to be oriented iff $\bar{\exists} A_{y_{1}, y_{2}, \ldots, y_{r}}=$ $1 A_{y_{1}, y_{2}, \ldots, y_{r}} \prec \prec$ area $A_{x_{1}, x_{2}, \ldots, x_{r}}$ and $\exists x_{i}, i \in(1,2, \ldots, r), \forall A_{y_{1}, \ldots, x_{i}, \ldots, y_{r}} A_{y_{1}, \ldots, x_{i}, \ldots, y_{r}}=$ 0 .

where $x_{i}$ is the coordinate of the domain to be oriented. According to the above definition, the domains to be oriented in Fig. [6] are Area $_{2,0}$, Area $_{2,1}$ and $A r e a_{4,0}$.

The domain to be oriented needs a corresponding oriented operation. We design the recombination operator as follows. Let $U=\left(u_{1}, u_{2}, \ldots, u_{n}\right)$ and $V=\left(v_{1}, v_{2}, \ldots, v_{n}\right)$ represent the parent individuals for recombination and $n$ is the dimension of the decision space. Then, the offspring is defined as $W=$ $\left(w_{1}, w_{2}, \ldots, w_{n}\right) ; w_{i}=a\left(u_{i}-v_{i}\right)+v_{i}$, where $a$ is a random number between 0 to 1 . It is not hard to find that $w_{i}$ is located between $u_{i}$ and $v_{i}$, because most of the multiobjective optimization problems meet the connectivity [40], that is to say, the solutions that are distributed like neighborhood in the decision space will be also distributed like neighborhood when mapped to the objective space. Therefore, the new generated individual is more likely located in the area between $U$ and $V$. In addition, we select the individual in the unit domain which is nearest to the domain to be oriented with a larger probability for recombination. For the domain which only has individuals at one end, such as Area $_{4,0}$ in Fig. 6, we select the individual in the unit domain which is nearest to the domain to be oriented and the individual in the other unit domain to recombine.

\subsection{Environmental Guiding Mechanism}

The dynamic environmental guiding mechanism refers to guiding the different sub-populations to evolve toward their desired environments based on the new environmental knowledge and regulation, so that the population diversity is enhanced. Similarly, we use the recombination operator introduced in Section $\mathrm{E}$, but $a$ is a random number between 0.8 to 1 . For different sub-populations, the strategy to select parent individuals to be recombined is different: 
For the individual sub1_indiv that wants to go back in subpopulation1, firstly, we need to calculate which unit domain coordinates of guide-individuals are located between sub1_indiv.old_area $a_{i}$ and sub1_indiv.new_area $a_{i}$, and then we select the individual that is the closest to sub1_indiv.old_area $a_{i}$. If multiple individuals are in the same unit domain, the representative individual in the unit domain is selected.

For the individual sub2_indiv that does not want to make any change in subpopulation2, a recombination operation is not needed.

For the individual sub3_indiv that does not want to go back in subpopulation3: if sub3_indiv.old_area ${ }_{i}>$ sub3_indiv.new_area ${ }_{i}$, we need to calculate which unit domain coordinates of guide-individuals are greater than sub3_indiv.old_area ${ }_{i}$, and then select the individual that is the farthest to sub3_indiv.old_area ${ }_{i}$. If sub3_indiv.old_area $a_{i}<$ sub3_indiv.new_area ${ }_{i}$, we need to calculate which unit domain coordinates of guide-individuals are less than sub3_indiv.old_area $a_{i}$, and then select the individual that is the farthest to sub3_indiv.old_area ${ }_{i}$. Similarly, if multiple individuals are in the same unit domain, the representative individual in the unit domain is selected.

Fig. 7 is an example of recombination strategy. For individual A, the selected another parent individual for recombination is $\mathrm{A}^{*}$.

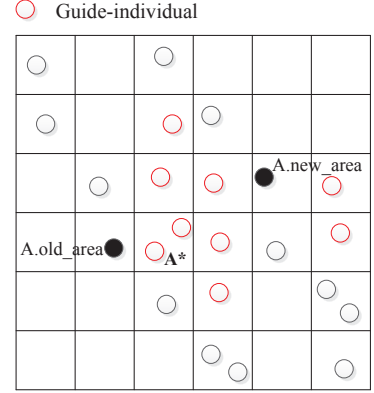

(a) Subpopulation1

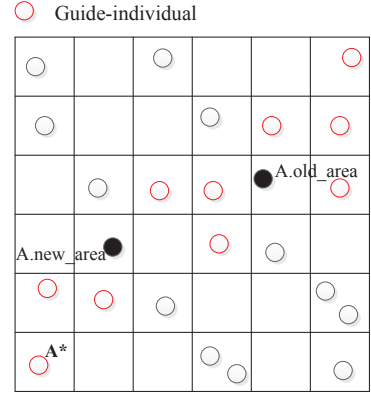

(b) Subpopulation3

A.old_area $_{i}>$ A.new_area $a_{i}$

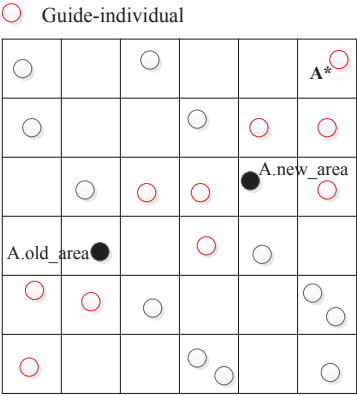

(c) Subpopulation3

A.old_area $_{i}<$ A.new_area

Figure 7: Example of different recombinations. 


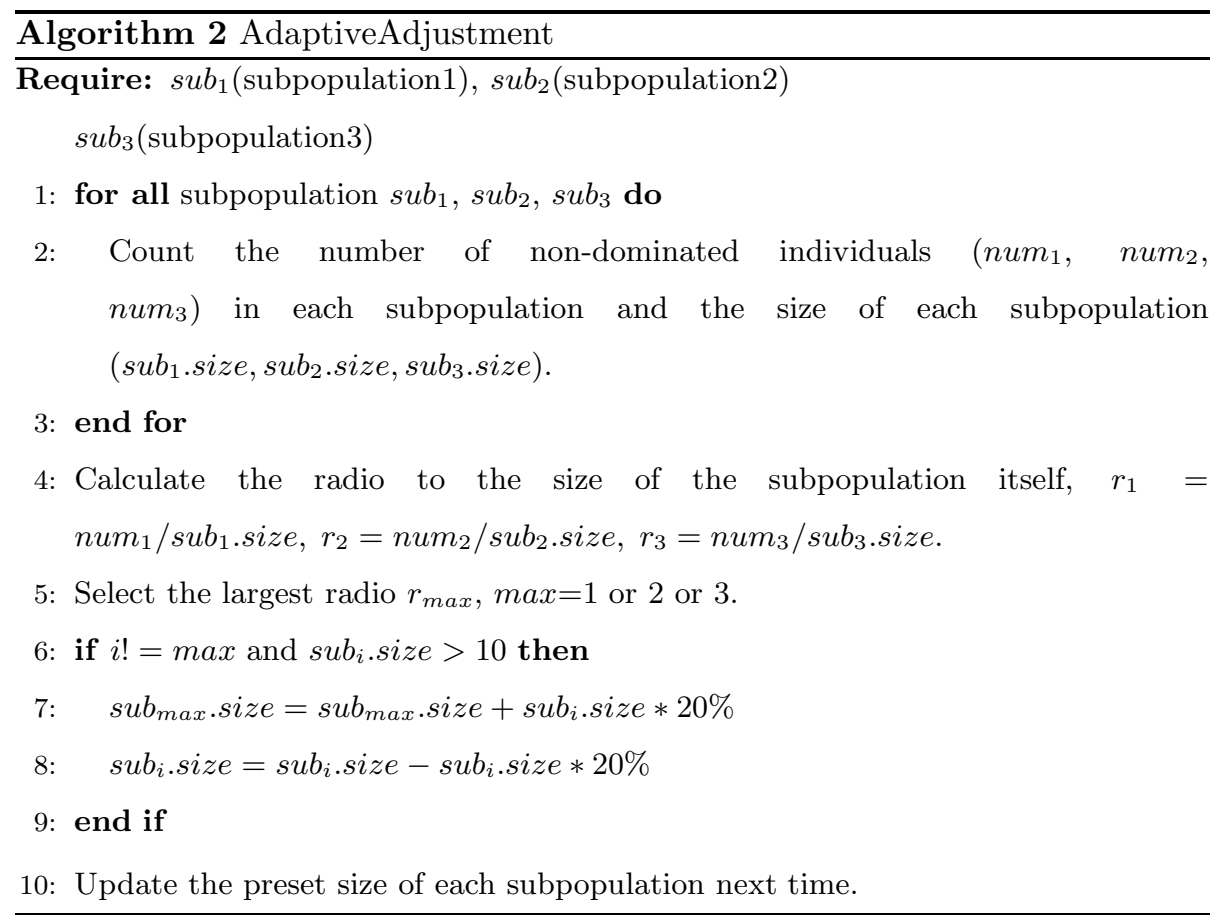

Meanwhile, in order to better solve some DMOPs with regular changes, the size of three sub-populations is adaptively adjusted. First, the combined population of the three sub-populations is evaluated. We count the non-dominated individuals, and then compare the ratio of number of non-dominated individuals in each sub-population to the size of the subpopulation. For the two subpopulations with smaller ratios, when the environment changes next time, the size of two sub-populations is reduced by $20 \%$, and no longer decreased until its size is less than 10. The size of the subpopulation with the largest ratio will increase accordingly. Algorithm 2 gives a detailed procedure of adaptive adjustment strategy.

In addition, for periodic DMOPs, we introduce the strategy of memory when the environment changes. We store the non-dominated individuals of the current population in the memory pool, non-dominated sort these stored individuals in the memory pool, and select Msize optimal individuals which adapt best to the new environment. When the size of memory pool is over twice of the population 


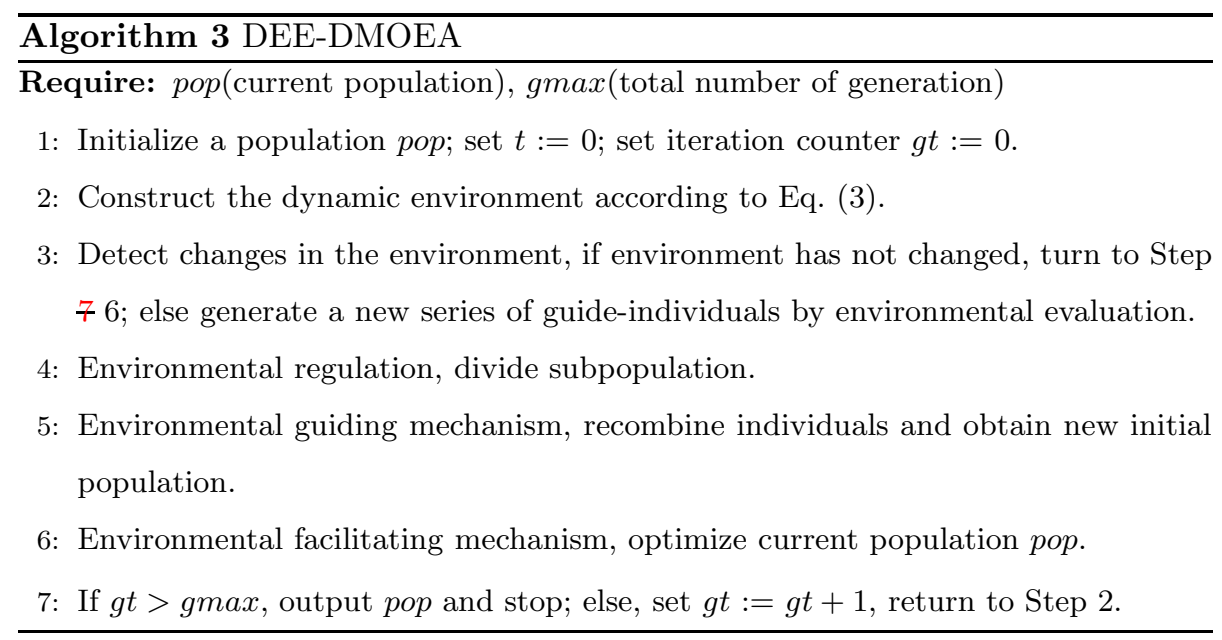

size, we use the principle of first in first out (FIFO) to update the memory pool, which ensures that the algorithm does not consume too much extra storage space and evaluation.

\subsection{The frame of DEE-DMOEA}

Now, we give the main procedure of DEE-DMOEA. The purpose of DEEDMOEA is to accelerate the convergence speed of the population at the static optimization phase and improve the convergence and distribution of the population. Meanwhile, it is to obtain new initial population after each environmental change, so that the new population can quickly respond to changes in the dynamic environment. The pseudo-code of DEE-DMOEA is presented in detail in Algorithm 3.

\section{Test Instances and Performance Metrics}

\subsection{Test Instances}

In this paper, a series of test problems proposed in [34] with linear or nonlinear correlation between design variables were selected of various DMOOP types 1] to compare the performance. Among them, F1 and F4 are from the FDA test suite [1], F2 and F3 are from the DMOP test suite [23], and F5-F10 are 
newly proposed in [34]. JY1 and JY5 are newly proposed in [51]. F1-F4 are linear correlation between the decision variables, while F5-F10 are nonlinear correlation between the decision variables. Especially, F9 and F10 are more complicated problems, and it is more difficult for an algorithm to converge on them. The details of the ten problems can be found in [34].

\subsection{Performance Metrics}

Some metrics have been designed for dynamic optimization [45, 47, 46]. In this paper, we introduce the dynamic generational distance (DGD) [23] and inverted generational distance (DIGD) 34] metrics for DMOPs. The DGD and DIGD metrics are defined as follows:

$$
\begin{gathered}
D G D=\frac{1}{|T|} \sum_{t \in T} G D\left(P F^{t}, P^{t}\right), \\
G D\left(P F^{t}, P^{t}\right)=\frac{\sum_{v \epsilon P^{t}} d\left(P F^{t}, v\right)}{\left|P^{t}\right|} \\
D I G D=\frac{1}{|T|} \sum_{t \in T} I G D\left(P F^{t}, P^{t}\right), \\
I G D\left(P F^{t}, P^{t}\right)=\frac{\sum_{v \epsilon P F^{t}} d\left(v, P^{t}\right)}{\left|P F^{t}\right|}
\end{gathered}
$$

where $P F^{t}$ is a set of uniformly distributed Pareto optimal points in the $P F$ at time $t$, and $P^{t}$ is the solutions obtained at time $t . d\left(P F^{t}, v\right)=\min _{u \in P F^{t}} \sqrt{\sum_{j=1}^{m}\left(f_{j}^{(u)}-f_{j}^{(v)}\right)^{2}}$

is the distance between $v$ and $P F^{t} ; d\left(v, P^{t}\right)=\min _{u \in P^{t}} \sqrt{\sum_{j=1}^{m}\left(f_{j}^{(v)}-f_{j}^{(u)}\right)^{2}}$ is the distance between $v$ and $P^{t} ; T$ is a set of discrete time points in a run and $|T|$ is the cardinality of $T$. DGD evaluates convergence of the algorithm. The lower the DGD value is, the better convergence the obtained solution set has. DIGD is a comprehensive metric to evaluate the convergence and distribution. A lower DIGD value means that solution set obtained has better convergence and distribution.

\section{Experiments}

In this section, DEE-DMOEA will be compared to three other algorithms: the dynamic cooperative-competitive evolutionary algorithm (dCOEA), pro- 
posed by Goh and Tan [23], the population prediction strategy using optimization algorithm RM-MEDA [43] (PPS-RM), proposed by Zhou et al. 34], and the diversity maintenance on prediction, proposed by Ruan et al. [50]. In DEEDMOEA, the number of unit domain on each dimension is 40 ; the number of guide-individual is 100; the number of selected optimal individuals from memory pool $M$ size $=5$ (three objectives: 10$)$. The population size $N=100$ (three objectives: 200); frequency of change $\tau_{T}=25$; severity of change $n_{T}=10$. Other parameter settings of the three strategies use the given settings in [23] and [34].

Since the DEE-DMOEA in this paper needs to consume one time of evaluation in generating guide-individuals and memory pool. To be fair, the algorithm iterations require removing the number of evaluations consumed at every environmental change, and reducing the corresponding number of iterations. Therefore, the frequency of change is set to be $\tau_{T}=23$ in DEE-DMOEA. We ran each algorithm 20 times for each test instance independently. Each simulation ran for 2500 generations (DEE-DMOEA: 2300 generations) and each strategy was tracked to 100 times of environmental changes. As the dynamic test problems introduced in Section 5 are all period, according to the parameter setting of $n_{T}$, the environment will change periodically with unequal frequency ranging from 2 to 40. So, in order to discuss the performances of different strategies in each period, the result of the experiment is divided into three stages except for the first environmental change. Each stage tracks to 33 times of environmental changes and its average is taken as the result. The statistical results of DIGD and DGD over 20 runs can be found in Table 1.

\subsection{Comparative Study}

As can be seen in Table 1, in terms of comprehensive evaluation, DEEDMOEA performs better than dCOEA, PPS-RM and DMS on most of the test problems; the mean DIGD in each stage is the smallest and becomes more and more stable. Especially, in the first stage, the metric values are significantly better than the other three algorithms. On F1-F4, where the decision variables 
Table 1: Statistical results of DIGD and DGD metric for four algorithms

\begin{tabular}{|c|c|c|c|c|c|c|c|c|c|}
\hline \multirow{2}{*}{ Problems } & \multirow{2}{*}{ Statistic } & \multicolumn{4}{|c|}{ DGD } & \multicolumn{4}{|c|}{ DIGD } \\
\hline & & dCOEA & PPS-RM & DMS & DEE-DMOEA & dCOEA & PPS-RM & DMS & DEE-DMOEA \\
\hline \multirow{4}{*}{ F1 } & Mean & $2.01 \mathrm{E}-3$ & $4.92 \mathrm{E}-2$ & $6.90 \mathrm{E}-3$ & $2.56 \mathrm{E}-3$ & $1.75 \mathrm{E}-2$ & $3.17 \mathrm{E}-2$ & $7.90 \mathrm{E}-3$ & $4.78 \mathrm{E}-3$ \\
\hline & 1st stage & $2.47 \mathrm{E}-3$ & $1.38 \mathrm{E}-1$ & $9.00 \mathrm{E}-3$ & $2.99 \mathrm{E}-3$ & $1.78 \mathrm{E}-2$ & $8.26 \mathrm{E}-2$ & $9.60 \mathrm{E}-3$ & $5.08 \mathrm{E}-3$ \\
\hline & 2nd stage & $1.71 \mathrm{E}-3$ & $4.93 \mathrm{E}-3$ & $7.20 \mathrm{E}-3$ & $2.43 \mathrm{E}-3$ & $1.75 \mathrm{E}-2$ & $6.32 \mathrm{E}-3$ & $7.20 \mathrm{E}-3$ & $4.66 \mathrm{E}-3$ \\
\hline & 3rd stage & $1.86 \mathrm{E}-3$ & $4.85 \mathrm{E}-3$ & $5.80 \mathrm{E}-3$ & $2.25 \mathrm{E}-3$ & $1.71 \mathrm{E}-2$ & $6.28 \mathrm{E}-3$ & $7.10 \mathrm{E}-3$ & $4.60 \mathrm{E}-3$ \\
\hline \multirow{4}{*}{$\mathrm{F} 2$} & Mean & $7.95 \mathrm{E}-4$ & $5.17 \mathrm{E}-3$ & $9.40 \mathrm{E}-3$ & $8.24 \mathrm{E}-4$ & $1.19 \mathrm{E}-2$ & $7.72 \mathrm{E}-3$ & $1.29 \mathrm{E}-2$ & $3.88 \mathrm{E}-3$ \\
\hline & 1st stage & $7.90 \mathrm{E}-4$ & $7.65 \mathrm{E}-3$ & $2.12 \mathrm{E}-2$ & $9.54 \mathrm{E}-4$ & $1.19 \mathrm{E}-2$ & $1.17 \mathrm{E}-2$ & $2.81 \mathrm{E}-2$ & $3.98 \mathrm{E}-3$ \\
\hline & 2nd stage & $7.84 \mathrm{E}-4$ & $3.92 \mathrm{E}-3$ & $3.50 \mathrm{E}-3$ & $7.44 \mathrm{E}-4$ & $1.26 \mathrm{E}-2$ & $5.73 \mathrm{E}-3$ & $5.40 \mathrm{E}-3$ & $3.86 \mathrm{E}-3$ \\
\hline & 3rd stage & $8.11 \mathrm{E}-4$ & $3.93 \mathrm{E}-3$ & $3.50 \mathrm{E}-3$ & $7.73 \mathrm{E}-4$ & $1.12 \mathrm{E}-2$ & $5.66 \mathrm{E}-3$ & $5.40 \mathrm{E}-3$ & $3.82 \mathrm{E}-3$ \\
\hline \multirow{4}{*}{ F3 } & Mean & $2.48 \mathrm{E}-3$ & $7.29 \mathrm{E}-2$ & $7.80 \mathrm{E}-3$ & $2.51 \mathrm{E}-3$ & $2.16 \mathrm{E}-2$ & $5.68 \mathrm{E}-2$ & $9.10 \mathrm{E}-3$ & $4.91 \mathrm{E}-3$ \\
\hline & 1st stage & $3.23 \mathrm{E}-3$ & $2.09 \mathrm{E}-1$ & $1.12 \mathrm{E}-2$ & $3.30 \mathrm{E}-3$ & $2.54 \mathrm{E}-2$ & $1.58 \mathrm{E}-1$ & $1.29 \mathrm{E}-2$ & $5.49 \mathrm{E}-3$ \\
\hline & 2nd stage & $1.91 \mathrm{E}-3$ & $4.56 \mathrm{E}-3$ & $6.10 \mathrm{E}-3$ & $2.46 \mathrm{E}-3$ & $1.98 \mathrm{E}-2$ & $6.17 \mathrm{E}-3$ & $7.10 \mathrm{E}-3$ & $4.89 \mathrm{E}-3$ \\
\hline & 3rd stage & $2.30 \mathrm{E}-3$ & $4.62 \mathrm{E}-3$ & $5.90 \mathrm{E}-3$ & $1.78 \mathrm{E}-3$ & $1.97 \mathrm{E}-2$ & $6.15 \mathrm{E}-3$ & $7.10 \mathrm{E}-3$ & $4.35 \mathrm{E}-3$ \\
\hline \multirow{4}{*}{ F4 } & Mean & $3.05 \mathrm{E}-2$ & $1.11 \mathrm{E}-1$ & $1.10 \mathrm{E}-1$ & $1.75 \mathrm{E}-2$ & $5.38 \mathrm{E}-2$ & $9.13 \mathrm{E}-2$ & $1.08 \mathrm{E}-1$ & $4.90 \mathrm{E}-2$ \\
\hline & 1st stage & $2.57 \mathrm{E}-2$ & $1.33 \mathrm{E}-1$ & $1.30 \mathrm{E}-1$ & $2.17 \mathrm{E}-2$ & $5.60 \mathrm{E}-2$ & $9.76 \mathrm{E}-2$ & $1.16 \mathrm{E}-1$ & $5.16 \mathrm{E}-2$ \\
\hline & 2nd stage & $3.66 \mathrm{E}-2$ & $9.74 \mathrm{E}-2$ & $1.10 \mathrm{E}-1$ & $1.60 \mathrm{E}-2$ & $5.22 \mathrm{E}-2$ & $8.48 \mathrm{E}-2$ & $1.04 \mathrm{E}-1$ & $4.78 \mathrm{E}-2$ \\
\hline & 3rd stage & $2.92 \mathrm{E}-2$ & $1.03 \mathrm{E}-1$ & $1.70 \mathrm{E}-1$ & $1.50 \mathrm{E}-2$ & $5.33 \mathrm{E}-2$ & $8.78 \mathrm{E}-2$ & $1.04 \mathrm{E}-1$ & $4.77 \mathrm{E}-2$ \\
\hline \multirow{4}{*}{ F5 } & Mean & $2.23 \mathrm{E}-1$ & $1.86 \mathrm{E}-1$ & $2.40 \mathrm{E}-2$ & $2.38 \mathrm{E}-2$ & $3.33 \mathrm{E}-1$ & $7.62 \mathrm{E}-2$ & $1.50 \mathrm{E}-2$ & $1.93 \mathrm{E}-2$ \\
\hline & 1st stage & $4.13 \mathrm{E}-1$ & $5.20 \mathrm{E}-1$ & $3.90 \mathrm{E}-2$ & $3.61 \mathrm{E}-2$ & $5.54 \mathrm{E}-1$ & $1.92 \mathrm{E}-1$ & $1.80 \mathrm{E}-2$ & $2.64 \mathrm{E}-2$ \\
\hline & 2nd stage & $1.59 \mathrm{E}-1$ & $2.25 \mathrm{E}-2$ & $3.00 \mathrm{E}-2$ & $2.09 \mathrm{E}-2$ & $2.58 \mathrm{E}-1$ & $2.07 \mathrm{E}-2$ & $1.38 \mathrm{E}-2$ & $1.77 \mathrm{E}-2$ \\
\hline & 3rd stage & $9.83 \mathrm{E}-2$ & $1.56 \mathrm{E}-2$ & $1.80 \mathrm{E}-2$ & $1.44 \mathrm{E}-2$ & $1.87 \mathrm{E}-1$ & $1.59 \mathrm{E}-2$ & $1.33 \mathrm{E}-2$ & $1.39 \mathrm{E}-2$ \\
\hline \multirow{4}{*}{ F6 } & Mean & $1.15 \mathrm{E}-1$ & $8.90 \mathrm{E}-2$ & $3.50 \mathrm{E}-2$ & $1.57 \mathrm{E}-2$ & $2.51 \mathrm{E}-1$ & $3.95 \mathrm{E}-2$ & $2.35 \mathrm{E}-2$ & $1.36 \mathrm{E}-2$ \\
\hline & 1st stage & $1.62 \mathrm{E}-1$ & $2.36 \mathrm{E}-1$ & $8.80 \mathrm{E}-2$ & $2.11 \mathrm{E}-2$ & $3.71 \mathrm{E}-1$ & $8.77 \mathrm{E}-2$ & $5.16 \mathrm{E}-2$ & $1.64 \mathrm{E}-2$ \\
\hline & 2nd stage & $8.32 \mathrm{E}-2$ & $1.85 \mathrm{E}-2$ & $9.00 \mathrm{E}-3$ & $1.38 \mathrm{E}-2$ & $1.92 \mathrm{E}-1$ & $1.77 \mathrm{E}-2$ & $9.40 \mathrm{E}-3$ & $1.28 \mathrm{E}-2$ \\
\hline & 3rd stage & $9.90 \mathrm{E}-2$ & $1.26 \mathrm{E}-2$ & $9.50 \mathrm{E}-3$ & $1.21 \mathrm{E}-2$ & $1.90 \mathrm{E}-1$ & $1.31 \mathrm{E}-2$ & $9.60 \mathrm{E}-3$ & $1.16 \mathrm{E}-2$ \\
\hline \multirow{4}{*}{ F7 } & Mean & $9.42 \mathrm{E}-2$ & $1.68 \mathrm{E}-1$ & $2.80 \mathrm{E}-2$ & $1.87 \mathrm{E}-2$ & $2.19 \mathrm{E}-1$ & $5.42 \mathrm{E}-2$ & $1.66 \mathrm{E}-2$ & $1.42 \mathrm{E}-2$ \\
\hline & 1st stage & $1.24 \mathrm{E}-1$ & $4.79 \mathrm{E}-1$ & $6.90 \mathrm{E}-2$ & $2.41 \mathrm{E}-2$ & $3.17 \mathrm{E}-1$ & $1.36 \mathrm{E}-1$ & $3.37 \mathrm{E}-2$ & $1.77 \mathrm{E}-2$ \\
\hline & 2nd stage & $7.69 \mathrm{E}-2$ & $1.41 \mathrm{E}-2$ & $6.90 \mathrm{E}-3$ & $1.50 \mathrm{E}-2$ & $1.83 \mathrm{E}-1$ & $1.41 \mathrm{E}-2$ & $8.10 \mathrm{E}-3$ & $1.29 \mathrm{E}-2$ \\
\hline & 3rd stage & $8.17 \mathrm{E}-2$ & $1.21 \mathrm{E}-2$ & $7.00 \mathrm{E}-3$ & $1.70 \mathrm{E}-2$ & $1.58 \mathrm{E}-1$ & $1.26 \mathrm{E}-2$ & $8.20 \mathrm{E}-3$ & $1.20 \mathrm{E}-2$ \\
\hline \multirow{4}{*}{ F8 } & Mean & $1.08 \mathrm{E}+0$ & $1.21 \mathrm{E}+0$ & $1.70 \mathrm{E}-1$ & $1.53 \mathrm{E}-1$ & $2.58 \mathrm{E}-1$ & $3.83 \mathrm{E}-1$ & $1.19 \mathrm{E}-1$ & $9.15 \mathrm{E}-2$ \\
\hline & 1st stage & $9.84 \mathrm{E}-1$ & $1.55 \mathrm{E}+0$ & $1.90 \mathrm{E}-1$ & $2.32 \mathrm{E}-1$ & $2.98 \mathrm{E}-1$ & $4.64 \mathrm{E}-1$ & $1.25 \mathrm{E}-1$ & $1.08 \mathrm{E}-1$ \\
\hline & 2nd stage & $1.08 \mathrm{E}+0$ & $9.94 \mathrm{E}-1$ & $1.60 \mathrm{E}-1$ & $1.11 \mathrm{E}-1$ & $2.46 \mathrm{E}-1$ & $3.22 \mathrm{E}-1$ & $1.16 \mathrm{E}-1$ & $8.17 \mathrm{E}-2$ \\
\hline & 3rd stage & $1.19 \mathrm{E}+0$ & $1.10 \mathrm{E}+0$ & $1.70 \mathrm{E}-1$ & $1.17 \mathrm{E}-1$ & $2.29 \mathrm{E}-1$ & $3.62 \mathrm{E}-1$ & $1.17 \mathrm{E}-1$ & $8.42 \mathrm{E}-2$ \\
\hline \multirow{4}{*}{ F9 } & Mean & $9.76 \mathrm{E}-2$ & $4.25 \mathrm{E}-1$ & $5.50 \mathrm{E}-02$ & $4.17 \mathrm{E}-2$ & $2.00 \mathrm{E}-1$ & $2.38 \mathrm{E}-1$ & $3.30 \mathrm{E}-2$ & $2.91 \mathrm{E}-2$ \\
\hline & 1st stage & $1.34 \mathrm{E}-1$ & $1.04 \mathrm{E}+0$ & $4.90 \mathrm{E}-2$ & $8.85 \mathrm{E}-2$ & $3.07 \mathrm{E}-1$ & $5.65 \mathrm{E}-1$ & $3.06 \mathrm{E}-2$ & $5.91 \mathrm{E}-2$ \\
\hline & 2nd stage & $7.70 \mathrm{E}-2$ & $1.20 \mathrm{E}-1$ & $6.40 \mathrm{E}-2$ & $2.25 \mathrm{E}-2$ & $1.60 \mathrm{E}-1$ & $7.11 \mathrm{E}-2$ & $3.67 \mathrm{E}-2$ & $1.60 \mathrm{E}-2$ \\
\hline & 3rd stage & $8.17 \mathrm{E}-2$ & $1.18 \mathrm{E}-1$ & $5.30 \mathrm{E}-2$ & $1.41 \mathrm{E}-2$ & $1.34 \mathrm{E}-1$ & $7.81 \mathrm{E}-2$ & $3.17 \mathrm{E}-2$ & $1.24 \mathrm{E}-2$ \\
\hline \multirow{4}{*}{ F10 } & Mean & $1.97 \mathrm{E}-1$ & $5.56 \mathrm{E}-1$ & $8.30 \mathrm{E}-2$ & $1.70 \mathrm{E}-1$ & $2.14 \mathrm{E}-1$ & $2.43 \mathrm{E}-1$ & $2.24 \mathrm{E}-1$ & $7.80 \mathrm{E}-2$ \\
\hline & 1st stage & $2.69 \mathrm{E}-1$ & $9.02 \mathrm{E}-1$ & $2.80 \mathrm{E}-1$ & $3.54 \mathrm{E}-1$ & $3.00 \mathrm{E}-1$ & $3.91 \mathrm{E}-1$ & $2.35 \mathrm{E}-1$ & $1.47 \mathrm{E}-1$ \\
\hline & 2nd stage & $1.73 \mathrm{E}-1$ & $3.91 \mathrm{E}-1$ & $3.80 \mathrm{E}-2$ & $1.20 \mathrm{E}-1$ & $1.83 \mathrm{E}-1$ & $1.75 \mathrm{E}-1$ & $2.16 \mathrm{E}-1$ & $6.41 \mathrm{E}-2$ \\
\hline & 3rd stage & $1.48 \mathrm{E}-1$ & $3.75 \mathrm{E}-1$ & $3.40 \mathrm{E}-2$ & $3.71 \mathrm{E}-2$ & $1.59 \mathrm{E}-1$ & $1.64 \mathrm{E}-1$ & $1.26 \mathrm{E}-1$ & $2.24 \mathrm{E}-2$ \\
\hline \multirow{4}{*}{ JY1 } & Mean & $8.80 \mathrm{E}-2$ & $1.00 \mathrm{E}-2$ & $3.80 \mathrm{E}-2$ & $4.10 \mathrm{E}-2$ & $1.82 \mathrm{E}-1$ & $1.86 \mathrm{E}-1$ & $3.16 \mathrm{E}-1$ & $1.82 \mathrm{E}-1$ \\
\hline & 1st stage & $9.30 \mathrm{E}-2$ & $2.30 \mathrm{E}-2$ & $2.40 \mathrm{E}-2$ & $5.90 \mathrm{E}-2$ & $5.81 \mathrm{E}-1$ & $1.88 \mathrm{E}-2$ & $3.50 \mathrm{E}-2$ & $5.49 \mathrm{E}-2$ \\
\hline & 2nd stage & $8.50 \mathrm{E}-2$ & $4.00 \mathrm{E}-3$ & $3.90 \mathrm{E}-2$ & $1.20 \mathrm{E}-2$ & $5.80 \mathrm{E}-1$ & $7.53 \mathrm{E}-3$ & $2.40 \mathrm{E}-2$ & $1.50 \mathrm{E}-2$ \\
\hline & 3rd stage & $8.90 \mathrm{E}-2$ & $3.90 \mathrm{E}-3$ & $3.80 \mathrm{E}-2$ & $9.30 \mathrm{E}-3$ & $5.80 \mathrm{E}-1$ & $7.35 \mathrm{E}-3$ & $5.60 \mathrm{E}-2$ & $1.29 \mathrm{E}-2$ \\
\hline \multirow{4}{*}{ JY5 } & Mean & $1.60 \mathrm{E}-1$ & $1.90 \mathrm{E}-1$ & $4.80 \mathrm{E}-1$ & $24.60 \mathrm{E}-1$ & $1.82 \mathrm{E}-1$ & $1.86 \mathrm{E}-1$ & $3.16 \mathrm{E}-1$ & $1.82 \mathrm{E}-1$ \\
\hline & 1st stage & $1.60 \mathrm{E}-1$ & $1.30 \mathrm{E}-1$ & $2.40 \mathrm{E}-1$ & $1.20 \mathrm{E}-1$ & $9.97 \mathrm{E}-2$ & $1.28 \mathrm{E}-1$ & $4.23 \mathrm{E}-1$ & $1.21 \mathrm{E}-1$ \\
\hline & 2nd stage & $2.10 \mathrm{E}-1$ & $2.50 \mathrm{E}-1$ & $3.60 \mathrm{E}-1$ & $2.30 \mathrm{E}-1$ & $2.40 \mathrm{E}-1$ & $2.45 \mathrm{E}-1$ & $3.65 \mathrm{E}-1$ & $2.30 \mathrm{E}-1$ \\
\hline & 3rd stage & $1.50 \mathrm{E}-1$ & $1.80 \mathrm{E}-1$ & $2.60 \mathrm{E}-1$ & $1.70 \mathrm{E}-1$ & $1.84 \mathrm{E}-1$ & $1.79 \mathrm{E}-1$ & $3.25 \mathrm{E}-1$ & $1.76 \mathrm{E}-1$ \\
\hline
\end{tabular}


are linearly correlated, the mean DIGD of dCOEA is less than that of PPS-RM, but in the later two stages, PPS-RM performs better than dCOEA. On F5F8, where the decision variables are nonlinearly correlated, dCOEA performs worse than PPS-RM, and with the environmental periodic changes and the accumulation of experience, PPS-RM will stabilize in the latter two stages and shows a gradual improved trend. On F9 and F10, which are more complicated problems, the performances of dCOEA and PPS-RM are not satisfactory. On JY1, DEE-DMOEA is the best for the mean value; PPS-RM is the best for the other stages. On JY5, DEE-DMOEA is the best for all the stages.

In terms of convergence evaluation, DEE-DMOEA shows the best performance on most test problems. On F1, F2 and F3 problems, the DGD of dCOEA is relatively average and slightly better than that of DEE-DMOEA. But overall, the metric values are similar to DEE-DMOEA. Similar to the results of comprehensive evaluation, dCOEA performs better on problems which have linear correlation between decision variables, and performs worse on problems which have nonlinear correlation between decision variables than PPS-RM.

It is not hard to explain the results. It is mainly because the environmental facilitating mechanism of DEE-DMOEA can accelerate the convergence speed of the population at the static optimization phase after environmental changes. Meanwhile, the mechanism can guide the individuals to evolve toward the domain to be oriented, thereby improving the convergence and distribution of the population. When a change is detected, the environmental guiding mechanism helps the population to respond quickly to new changes and generate a new initial population and accelerate the convergence of the algorithm to the new optimal solutions. $\mathrm{dCOEA}$ is a competitive-cooperative co-evolutionary algorithm; it generates new individuals by selecting representatives of different sub-populations and themselves to recombine and evaluate. This method can achieve good results in solving problems where the decision variables are linearly correlated. However, on solving problems which have nonlinear correlation between the decision variables, it is ineffective.

PPS-RM uses an autoregressive model (AR) to predict a new population 
center at the next time by storing the population center on a continuous time series. Meanwhile, it predicts a new population distribution of the next time by recording the shape of population in the last two moments. The algorithm relies on the periodic environmental changes and accumulation of experience, so at the initial stages, the performance is poor. Along with the periodic changes, the accumulation of historical information could be sufficient to better predict the initial population. Therefore, PPS-RM will stabilize in the latter two stages.

\subsection{Comparison of Distribution of Final Obtained Population}

In order to visually analyze the performance of each algorithm, we choose four typical test problems, F1, F3, F6, and F9, and draw the distribution of final obtained populations of four algorithms for solving them at different time, shown in Fig. 8 to Fig. 11 .

By comparison, the experimental results are similar to those in the previous section. The convergence and diversity of DEE-DMOEA are far better than dCOEA and PPS-RM at the beginning stages of environmental change, which indicates that DEE-DMOEA is able to respond to environmental changes more quickly and accurately. Furthermore, the convergence and diversity of PPS-RM is poor, indicating when the accumulation of information is insufficient, PPS-RM can not make accurate predictions. In the later stages of running, DEE-DMOEA is the same as PPS-RM, which has a better convergence and distribution, and is slightly better than PPS-RM on the nonlinear problems. Although dCOEA can obtain solutions with better convergence on the linear problems, the distribution of solutions is poor. When solving nonlinear problems, dCOEA can only obtain a few of the dominated individuals, which indicates that the algorithm is not suitable for solving such problems. As to the ability to solve the complicated problem F9, the advantage of DEE-DMOEA is more obvious. The three other algorithms can not achieve better convergence and distribution, while DEEDMOEA can more accurately track to new optimal solutions and obtain a Pareto optimal solution set with better convergence and distribution. It indicates that DEE-DMOEA is more suitable for solving complicated nonlinear problems than 

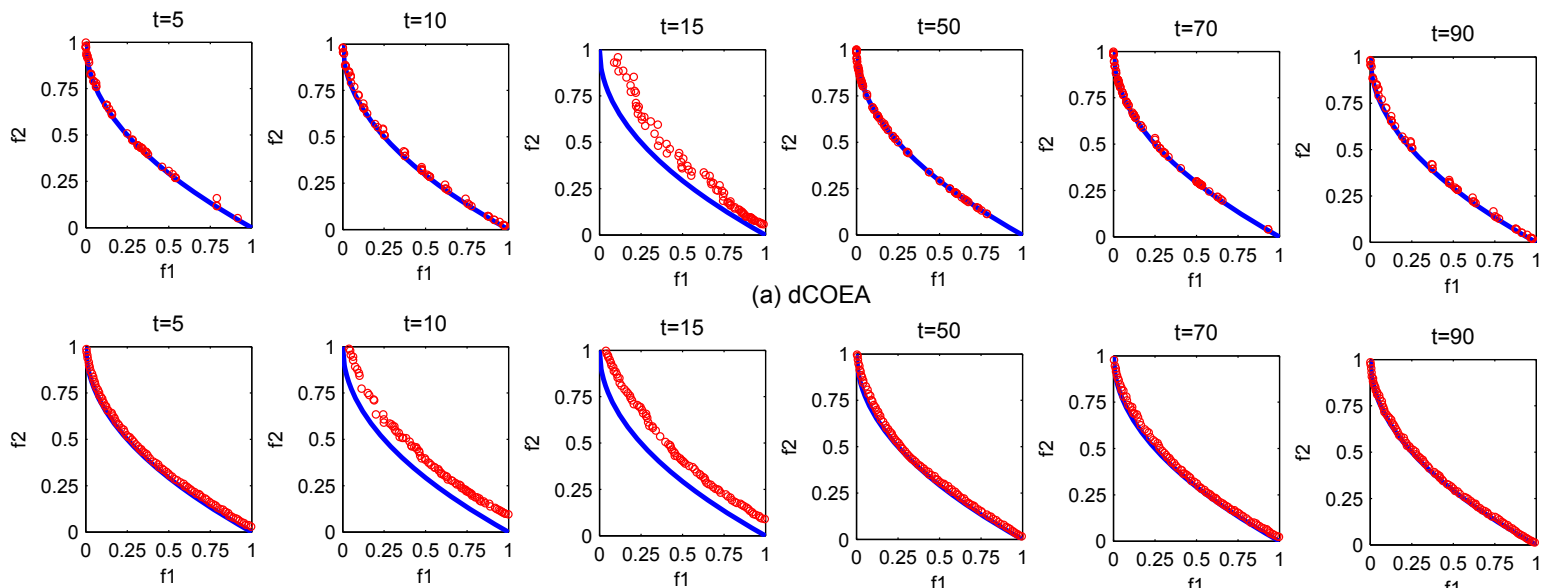

a) dCOEA
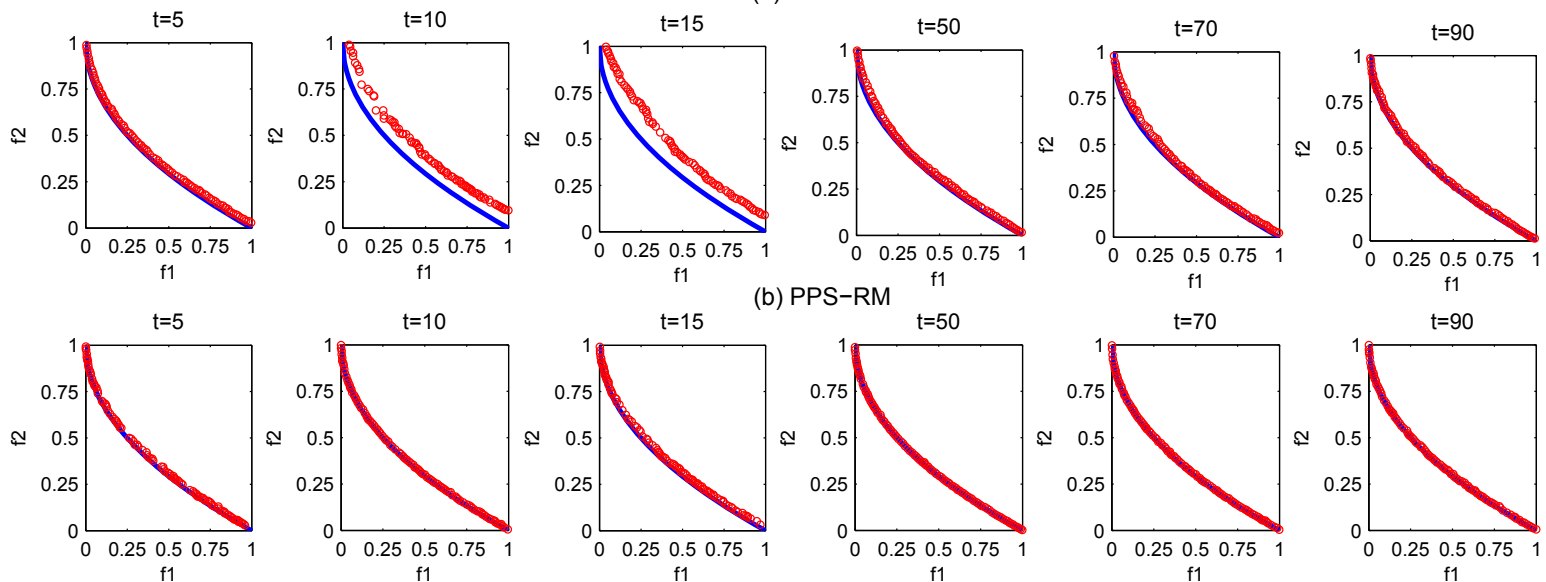

(b) PPS-RM $t=50$
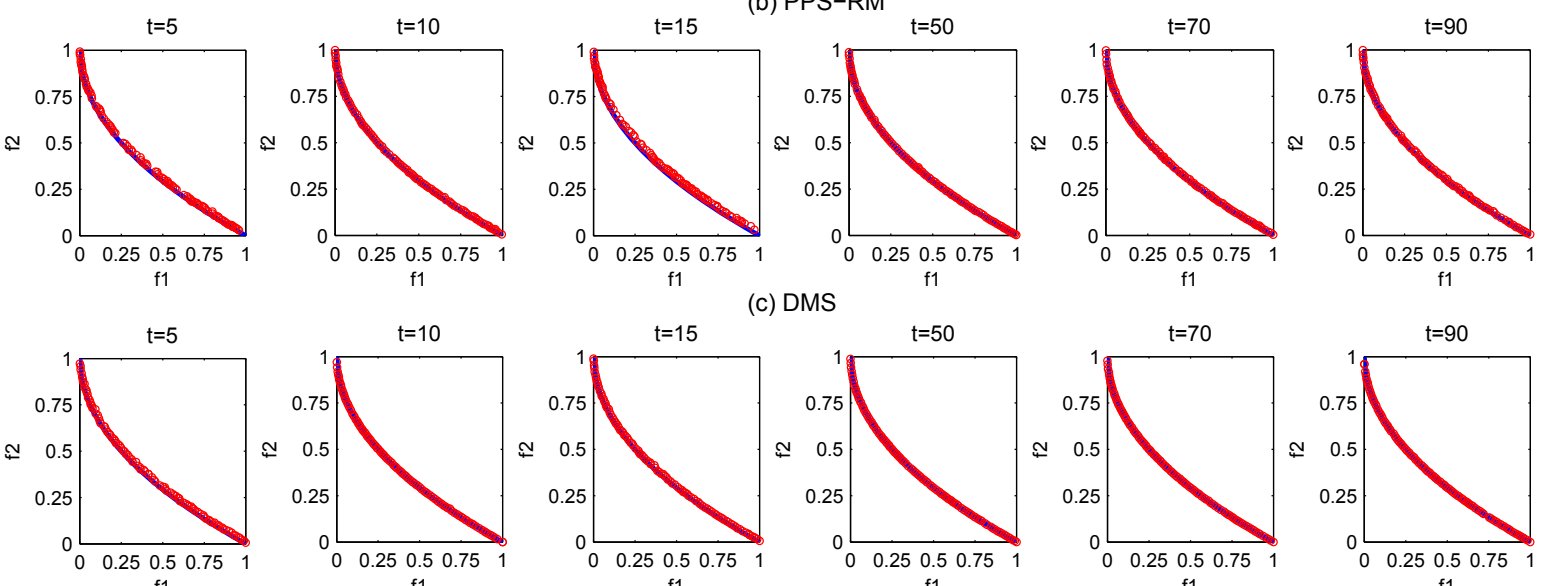

(c) DMS
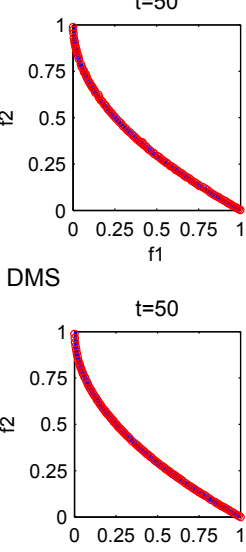

(d) DEE-DMOEA ${ }^{\mathrm{f}}$
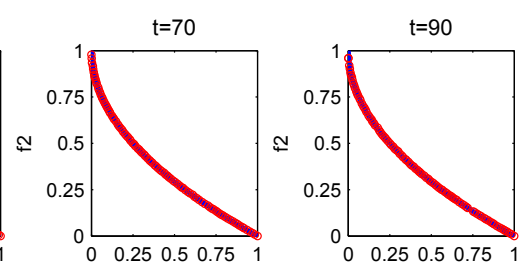

Figure 8: Solution sets for four algorithms at six different time steps on F1. 

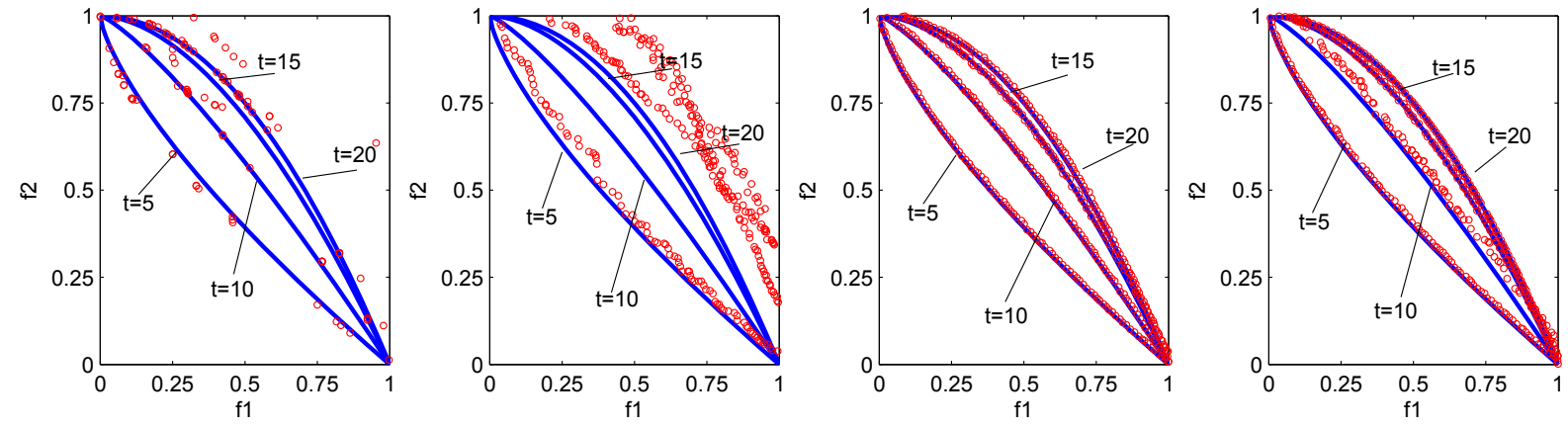

(a) dCOEA

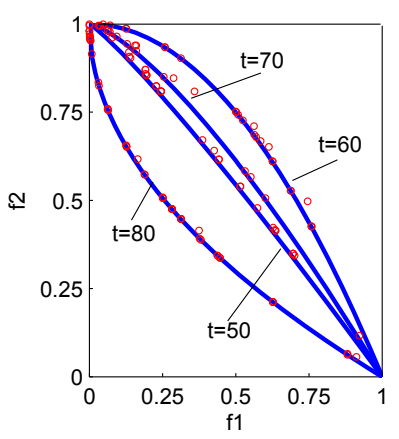

(b) PPS-RM

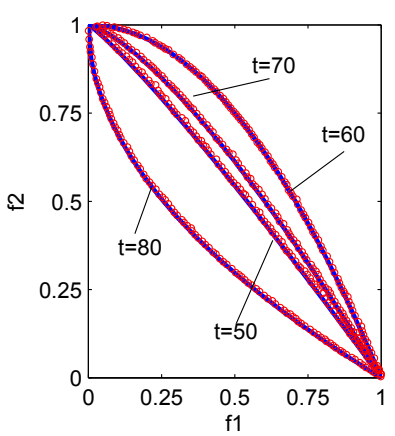

(a) dCOEA

(b) PPS-RM

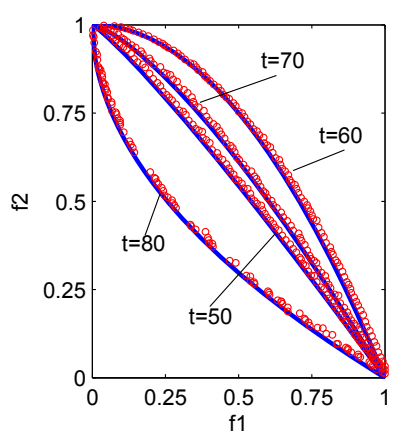

(c) DMS (d) DEE-DMOEA

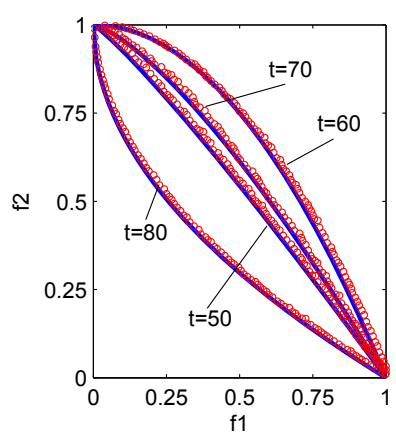

(d) DEE-DMOEA

Figure 9: Solution sets for four algorithms at eight different time steps on F3. 

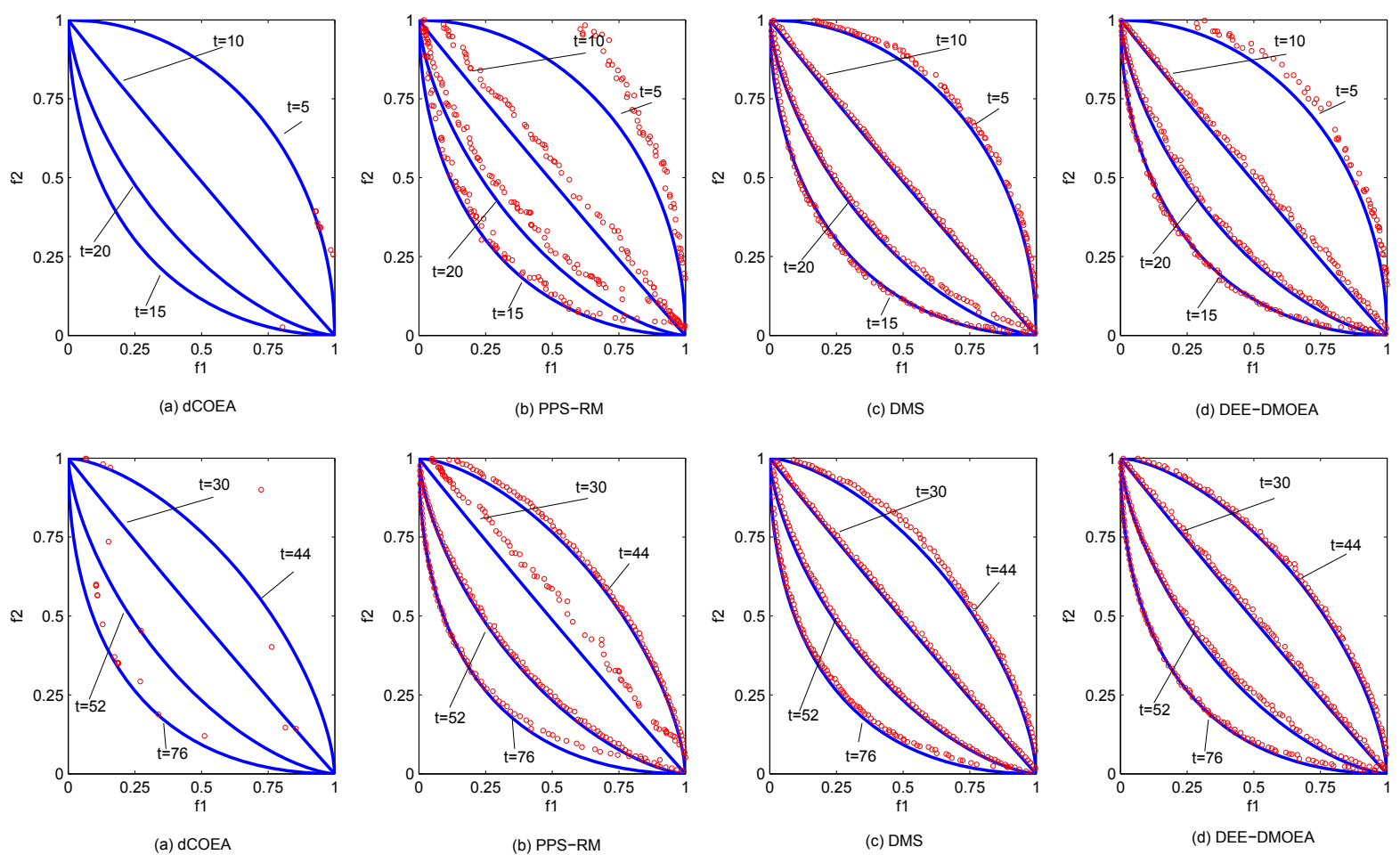

Figure 10: Solution sets for four algorithms at eight different time steps on F6. 

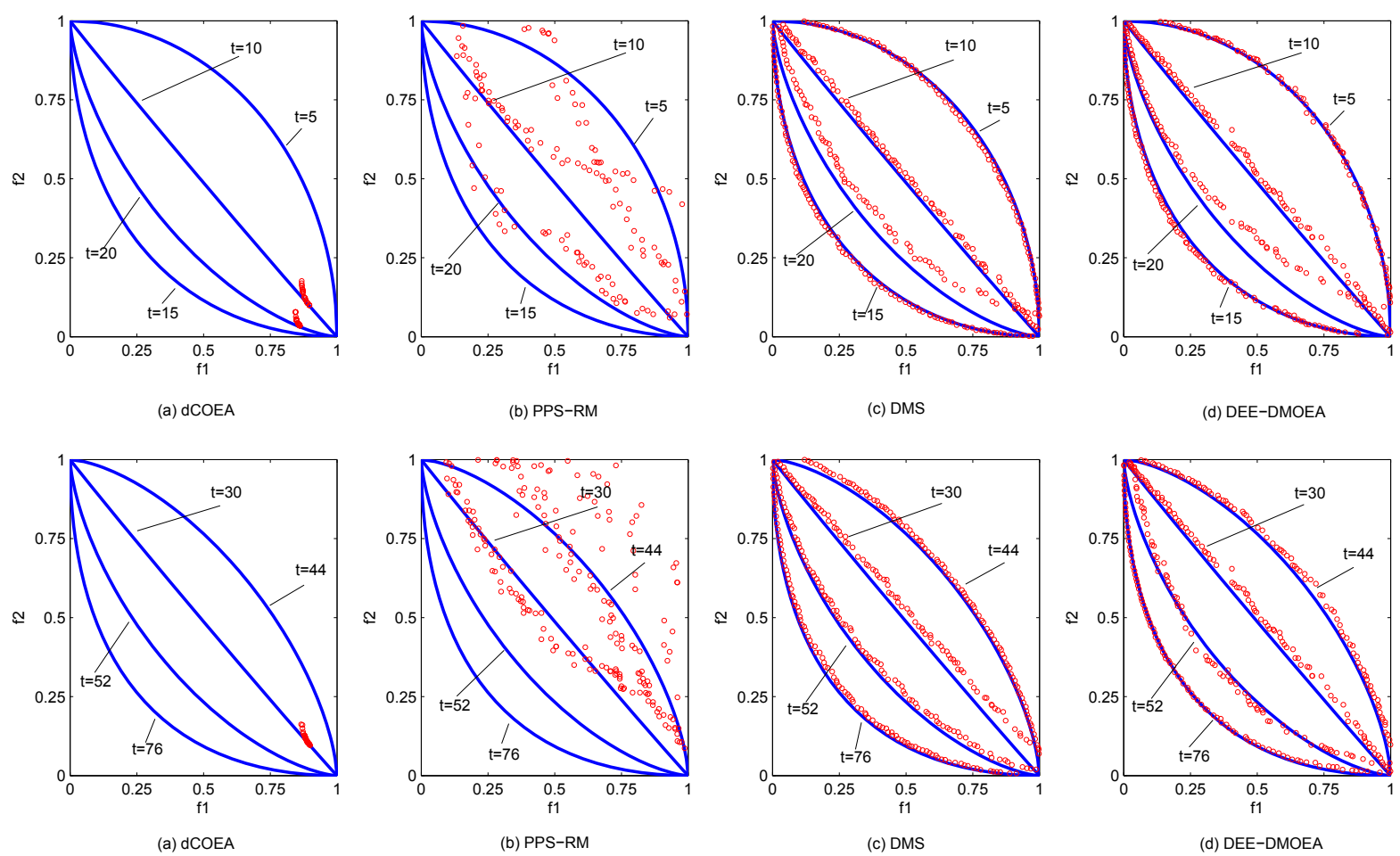

Figure 11: Solution sets for four algorithms at eight different time steps on F9. 

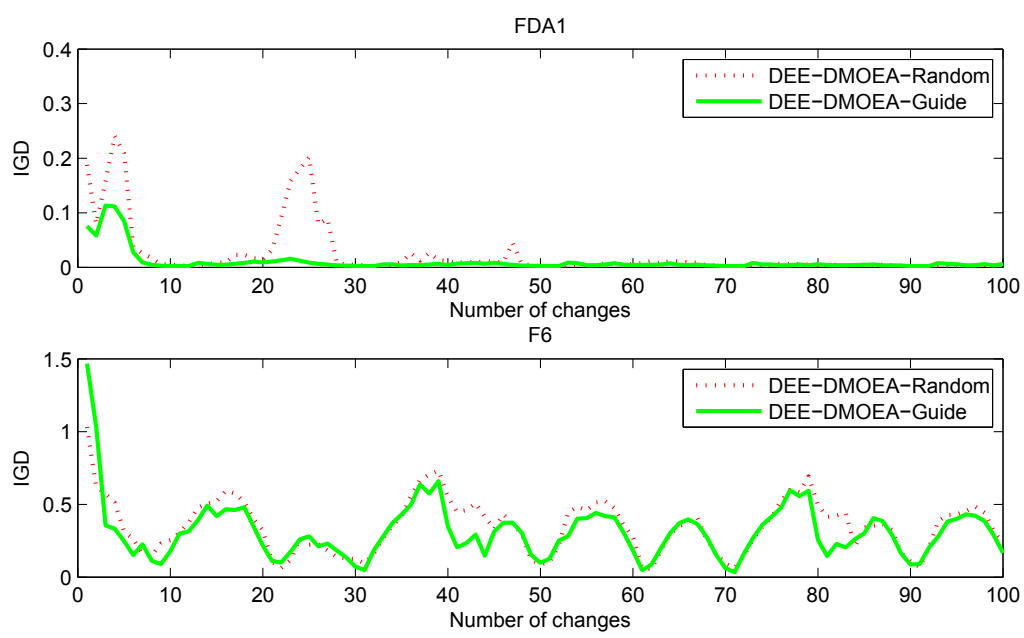

Figure 12: IGD trend comparison of DEE-dMOEA-Random and DEE-dMOEA-Guide over number of changes for 20 runs on FDA1 and F6.

the other three algorithms.

\subsection{Comparison of DEE-DMOEA-Guide and DEE-DMOEA-Random}

In Section 4.3 Environmental Evaluation, we generated some guide-individuals to guide evolution when evaluation was for population. For deeper observation of the role of the part, we use random individuals to replace the guide-individuals. The algorithm with random individuals is called DEE-dMOEA-Random, and the algorithm with guide-individuals is called DEE-dMOEA-Guide.

Fig. 12 shows the IGD trend comparison of DEE-dMOEA-Random and DEE-dMOEA-Guide over the number of changes for 20 runs on FDA1 and F6. On FDA1, it can be seen that the IGD graph of DEE-dMOEA-Guide is below the IGD graph of DEE-dMOEA-Random over most of the changes, especially in the early stage. On F6, the comparison result of IGD trend is similar to FDA1. However, the fluctuation on F6 is larger than FDA1. Overall, the effect of DEE-dMOEA-Guide is better than DEE-dMOEA-Random. 


\section{Conclusions}

In this paper, we have proposed a dynamic multiobjective evolutionary algorithm based on a dynamic environment evolutionary model (DEE-DMOEA) to solve dynamic multiobjective problems. In the proposed algorithm, we build a dynamic environment evolutionary model, which makes use of the dynamic environment to record different knowledge and information generated by population before and after an environmental change, and in turn, the knowledge and information guide the search in the dynamic environment. The model accelerates the convergence speed of population at the static optimization phase and improve the convergence and distribution of the population. Furthermore, it enhances population diversity by guided method when a change is detected, so that the new population can quickly respond to changes in the dynamic environment.

Compared with three other algorithms, DEE-DMOEA has shown faster response to the environmental changes than peer algorithms in solving linear or nonlinear problems, with its solution set having better convergence and diversity. Our future work will be designing a more accurate dynamic environment evolutionary model. Furthermore, our focus in the future will also be the applications of the dynamic multiobjective evolutionary algorithms in practical problems.

\section{Acknowledgement}

This work was supported by the research projects: the National Natural Science Foundation of China under Grant Nos. 61502408 and 61673331, the Education Department Major Project of Hunan Province under Grant No. 17A212615 the CERNET Innovation Project under Grant No. NGII20150302. 


\section{References}

[1] M. Farina, K. Deb, and P. Amato, "Dynamic multiobjective optimization problems: test cases, approximations, and applications," IEEE Trans. Evol. Comput., 2004, 8(5):425-442.

[2] J. Branke, "Evolutionary optimization in dynamic environments," Kluwer Academic Publishers, 2002.

[3] Y. Jin, and J. Branke, "Evolutionary optimization in uncertain environments-A survey," IEEE Trans. Evol. Comput., 2005, 9(3):303-317.

[4] T. T. Nguyen, S. Yang and J. Branke "Evolutionary dynamic optimization: A survey of the state of the art," Swarm and Evolutionary Computation, 2012, 6:1-24.

[5] M. Helbig, A. P. Engelbrecht, "Population-based metaheuristics for continuous boundary-constrained dynamic multi-objective optimisation problems," Swarm and Evolutionary Computation, Vol. 14, pp. 34-46, 2014.

[6] C. A. C. Coello, "20 Years of evolutionary multiobjective optimization: what has been done and what remains to be done," in Computational Intelligence: Principles and Practice, IEEE Computational Intelligence Society., 2006, pp. 73-88.

[7] C. A. C. Coello, D. A. van Veldhuizen, and G. B. Lamont, "Evolutionary algorithms for solving multiobjective problems," New York: Springer-Verlag, 2007.

[8] S. Yang, M. Li, X. Liu, and J. Zheng, "A grid-based evolutionary algorithm for many-objective optimization," IEEE Trans. Evol. Comput., vol. 17, no. 5, pp. 721-736, Oct. 2013.

[9] M. Li, S. Yang, and X. Liu, "Shift-Based density estimation for paretobased algorithms in many-objective optimization," IEEE Trans. Evol. Comput., 18(3): 348-365, June 2014. 
[10] Z. Avdagic, S. Konjicija, and S. Omanovic, "Evolutionary approach to solving non-stationary dynamic multiobjective problems," Foundations of Computational Intelligence Volume 3, ser. Studies in Computational Intelligence, A. Abraham, A.-E. Hassanien, P. Siarry, and A. Engelbrecht, Eds. Springer Berlin/Heidelberg, 2009, vol. 203, pp. 267-289.

[11] R. Shang, L. Jiao, Y. Ren, L. Li, and L. Wang, "Quantum immune clonal coevolutionary algorithm for dynamic multiobjective optimization," Soft Computing, 2013, 1-14.

[12] M. Greeff and A. P. Engelbrecht, "Solving dynamic multiobjective problems with vector evaluated particle swarm optimization," in Proc. IEEE Congr. Evol. Comput., 2008, 2922-2929.

[13] L. Huang, H. Suh and A. Abraham, "Dynamic multiobjective optimization based on membrane computing for control of time-varying unstable plants," Information Sciences, 2011. 181:2370-2391.

[14] A. Isaacs, V. Puttige, T. Ray, W. Smith, and S. Anavatti, "Development of a memetic algorithm for dynamic multiobjective optimization and its applications for online neural network modeling of UAVs," in International Joint Conference on Neural Networks (IJCNN 2008), 2008, pp. 548-554.

[15] R. Liu, W. Zhang, L. Jiao, F. Liu and J. Ma, "A sphere-dominance based preference immune-inspired algorithm for dynamic multiobjective optimization," in Genetic and Evolutionary Computation Conference (GECCO $\underline{2010)}, 2011,423-430$.

[16] J. Wei and Y. Wang, "Hyper rectangle search based particle swarm algorithm for dynamic constrained multiobjective optimization problems," in Proc. IEEE Congr. Evol. Comput (CEC2012)., 2012, 259-266.

[17] C. Liu and Y. Wang, "Multiobjective evolutionary algorithm for dynamic nonlinear constrained optimization problems," Journal of Systems Engineering and Electronics, vol. 20, no. 1, pp. 204-210, 2009. 
[18] C. Liu and Y. Wang, "New evolutionary algorithm for dynamic multiobjective optimization problems," in Evolutionary Computation: Theory and Algorithms, vol. LNCS 4221, 2006, pp. 889-892.

[19] C. R. B. Azevedo and A. F. R. Araujo, "Generalized immigration schemes for dynamic evolutionary multiobjective optimization," in Proc. IEEE Congr. Evol. Comput., 2011. 2033-2040.

[20] A. D. Manriquez, G. T. Pulido and J. G. R. Torres, "Handling dynamic multiobjective problems with particle swarm optimization," in 2nd International Conference on Agents and Artificial Intelligence (ICAART2010), 2010. 337-342.

[21] M. Camara, J. Ortega and F. de Toro, "Approaching dynamic multiobjective optimization problems by using parallel evolutionary algorithms," Advances in multiobjective Nature Inspired Computing, 2010. 272:63-86.

[22] B. Zheng, "A new dynamic multiobjective optimization evolutionary algorithm," in Third International Conference on Natural Computation (ICNC 2007), 2007, pp. 565-570.

[23] C. K. Goh and K. C. Tan, "A competitive-cooperative coevolutionary paradigm for dynamic multiobjective optimization," IEEE Trans. Evol. Comput., 2009. 13:103-127.

[24] C. K. Goh, and K. C. Tan, "Evolutionary multiobjective Optimization in Uncertain Environments: Issues and Algorithms," springer-Verlag, Berlin, 2009.

[25] K. Deb, U. V. Rao, and S. Karthik, "Dynamic multiobjective optimization and decision-making using modified NSGA-II- a case study on hydrothermal power scheduling," in Evolutionary Multi-Criterion Optimization (EMO 2007), 2007, LNCS 4403, pp. 803-817. 
[26] Z. Zhang, "Multiobjective optimization immune algorithm in dynamic environments and its application to greenhouse control," Applied Soft Computing, vol. 8, no. 2, pp. 959-971, 2008.

[27] X. Yao, Y. Liu, and G. Lin, "Evolutionary programming made faster," IEEE Trans. Evol. Comput., 3(2): 82-102, 1999.

[28] Y. Wang and B. Li, "Investigation of memory-based multiobjective optimization evolutionary algorithm in dynamic environment," in Proc. IEEE Congr. Evol. Comput., 2009, 630-637.

[29] A. Isaacs, V. Puttige, T. Ray, W. Smith, and S. Anavatti, "Development of a memetic algorithm for dynamic multiobjective optimization and its applications for online neural network modeling of UAVs," in International Joint Conference on Neural Networks (IJCNN 2008), 2008, pp. 548-554.

[30] S. Guan, Q. Chen, and W. Mo, "Evolving dynamic multiobjective optimization problems with objective replacement," Artificial Intelligence Review, vol. 23, pp. 267-293, 2005.

[31] I. Hatzakis, and D. Wallace, "Dynamic multiobjective optimization with evolutionary algorithms: A forward-looking approach," in Genetic and Evolutionary Computation Conference (GECCO 2006), 2006, 1201-1208.

[32] I. Hatzakis, and D. Wallace, "Topology of anticipatory populations for evolutionary dynamic multiobjective optimization," in 11th AIAA/ISSMO Multidisciplinary Analysis and Optimization Conference, 2006.

[33] A. Zhou, Y. Jin, Q. Zhang, B. Sendhoff and E. Tsang, "Prediction-based population re-initialization for evolutionary dynamic multiobjective optimization," in Evolutionary Multi-Criterion Optimization (EMO 2007), 2007.832-846.

[34] A. Zhou, Y. Jin, and Q. Zhang, "A population prediction strategy for evolutionary dynamic multiobjective optimization," IEEE Transactions on Cybernetics, 2013. 
[35] Y. Ma, R. Liu, and R. Shang, "A hybrid dynamic multiobjective immune optimization algorithm using prediction strategy and improved differential evolution crossover operator," Neural Information Processing, 2011, vol. LNCS 7063, pp. 435-444.

[36] Y. Wang and B. Li, "Multi-strategy ensemble evolutionary algorithm for dynamic multiobjective optimization," Memetic Computing, vol. 2, pp. 324, 2009.

[37] T. M. Blackwell and P. J. Bentley, "Dynamic search with charged swarms," in Genetic and Evolutionary Computation Conference (GECCO 2002), 2002.19-26.

[38] K. Deb, "Multi-Objective Optimization using Evolutionary Algorithms," John Wiley \& Sons, UK, Chichester, 2001.

[39] K. Deb, A. Sinha, and S. Kukkonen, "Multi-Objective Test Problems, Linkages, and Evolutionary Methodologies," in Genetic and Evolutionary Computation Conference (GECCO 2006), 2006: 1141-1148.

[40] P. Borges, and M. Hansen, "A basis for future successes in multiobjective combinatorial optimization," Technical Report IMM-REP-1998-8, Institute of Mathematical Modelling, Technical University of Denmark, March 1998.

[41] M. Helbig and A. P. Engelbrecht, "Archive management for dynamic multiobjective optimisation problems using vector evaluated particle swarm optimization," in Proc. IEEE Congr. Evol. Comput (CEC2011), 2011, pp. 2047-2054.

[42] W. T. Koo, C. K. Goh and K. C. Tan, "A predictive gradient strategy for multiobjective evolutionary algorithms in a fast changing environment," Memetic Computing, vol. 2, pp. 87-110, 2009.

[43] Q. Zhang, A. Zhou and Y. Jin, "RM-MEDA: A regularity model based multiobjective estimation of distribution algorithm," IEEE Trans. Evol. Comput., 2008, 12(1): 41-63. 
[44] K. Deb, A. Pratap, S. Agarwal, and T. Meyarivan, "A fast and elitist multiobjective genetic algorithm: NSGA-II," IEEE Trans. Evol. Comput., vol. 6, no. 2, pp. 182-197, 2002.

[45] M. Camara, J. Ortega, and F. de Toro, "Performance measures for dynamic multiobjective optimization," in Bio-Inspired Systems: Computational and Ambient Intelligence. Springer, 2009. 5517:760-767.

[46] E. Tantar, A. A. Tantar and P. Bouvry, "On dynamic multiobjective optimization classification and performance measures," in Proc. IEEE Congr. Evol. Comput (CEC2011)., 2011, 2759-2766.

[47] M. Helbig and A. Engelbrecht, "Issues with performance measures for dynamic multiobjective optimization," IEEE Symposium Series on Computational Intelligence, Singapore, April 2013, pp. 17-24.

[48] Z. Peng, J. Zheng, J. Zou, and M. Liu, "Novel prediction and memory strategies for dynamic multiobjective optimization," Soft Computing, vol. 19, no. 9, pp. 2633-2653, 2015.

[49] A. Zhou, Y. Jin, and Q. Zhang, "A population prediction strategy for evolutionary dynamic multiobjective optimization," IEEE Trans. on Cybern., vol. 44, no. 1, pp. 40-53, 2013.

[50] R. Gan, G. Yu, J. Zheng, J. Zou, and S. Yang. The effect of diversity maintenance on prediction in dynamic multi-objective optimization. Applied Soft Computing, 58: 631-647, 2017.

[51] S. Jiang and S. Yang, "Evolutionary dynamic multiobjective optimization: Benchmarks and algorithm comparisons". IEEE Trans. on Cybern., vol. 47, no. 1, pp. 198-211, January 2017. 